TRANSACTIONS OF THE

AMERICAN MATHEMATICAL SOCIETY

Volume 363, Number 10, October 2011, Pages 5293-5319

S 0002-9947(2011)05199-8

Article electronically published on May 20, 2011

\title{
DYNAMICAL PROPERTIES AND STRUCTURE OF JULIA SETS OF POSTCRITICALLY BOUNDED POLYNOMIAL SEMIGROUPS
}

\author{
RICH STANKEWITZ AND HIROKI SUMI
}

\begin{abstract}
We discuss the dynamic and structural properties of polynomial semigroups, a natural extension of iteration theory to random (walk) dynamics, where the semigroup $G$ of complex polynomials (under the operation of composition of functions) is such that there exists a bounded set in the plane which contains any finite critical value of any map $g \in G$. In general, the Julia set of such a semigroup $G$ may be disconnected, and each Fatou component of such $G$ is either simply connected or doubly connected. In this paper, we show that for any two distinct Fatou components of certain types (e.g., two doubly connected components of the Fatou set), the boundaries are separated by a Cantor set of quasicircles (with uniform dilatation) inside the Julia set of $G$. Important in this theory is the understanding of various situations which can and cannot occur with respect to how the Julia sets of the maps $g \in G$ are distributed within the Julia set of the entire semigroup $G$. We give several results in this direction and show how such results are used to generate (semi) hyperbolic semigroups possessing this postcritically boundedness condition.
\end{abstract}

\section{INTRODUCTION}

The dynamics of iteration of a complex analytic map has been studied quite deeply and in various contexts, e.g., rational, entire, and meromorphic maps. It is then natural to consider the generalization of this theory to the setting where the map may be changed at each point of the orbit, exactly as in a random walk. Instead of repeatedly applying the same map over and over again, one may start with a family of maps $\left\{h_{\lambda}: \lambda \in \Lambda\right\}$ and consider the dynamics of any iteratively defined composition sequence of maps, that is, any sequence $h_{\lambda_{n}} \circ \cdots \circ h_{\lambda_{1}}$ where each $\lambda_{k} \in \Lambda$. Assigning probabilities to the choice of map at each stage is the setting for research of random dynamics (see [9, 4, 6, 17, 5, 28, 30, 31, 32, 33. for previous work related to such dynamics). In this paper, however, we will concern ourselves with questions of dynamic stability, not just along such composition sequences one at a time, but rather when such stability exists no matter which composition sequence is chosen. Restricting one's attention to the case where all $h_{\lambda}$ are rational, one is led to study the dynamics of rational semigroups.

Received by the editors May 14, 2007 and, in revised form, August 13, 2009.

2010 Mathematics Subject Classification. Primary 37F10, 37F50, 30D05.

Key words and phrases. Complex dynamics, Julia sets, polynomial semigroups, random iteration, random complex dynamics.

The first author was partially supported by the BSU Lilly V grant. He would also like to thank Osaka University for their hospitality during his stay there while this work was begun.

(C)2011 American Mathematical Society Reverts to public domain 28 years from publication 
A rational semigroup is a semigroup generated by non-constant rational maps on the Riemann sphere $\overline{\mathbb{C}}$, with the semigroup operation being the composition of maps. We denote by $\left\langle h_{\lambda}: \lambda \in \Lambda\right\rangle$ the rational semigroup generated by the family of maps $\left\{h_{\lambda}: \lambda \in \Lambda\right\}$. A polynomial semigroup is a semigroup generated by non-constant polynomial maps. Research on the dynamics of rational semigroups was initiated by A. Hinkkanen and G.J. Martin in [11, who were interested in the role of the dynamics of polynomial semigroups while studying various onecomplex-dimensional moduli spaces for discrete groups. Also, F. Ren, Z. Gong, and W. Zhou studied such semigroups from the perspective of random dynamical systems (see [39, 10]). Note that there is a strong connection between the study of dynamics of rational semigroups and that of random complex dynamics (see 29, 30, 31, 32, 33]). For example, for a random dynamical system generated by a family of polynomials, we consider the function $T_{\infty}: \overline{\mathbb{C}} \rightarrow[0,1]$ of the probability of tending to $\infty$. It turns out that under certain conditions the function $T_{\infty}$ is continuous on $\overline{\mathbb{C}}$ and varies only on the Julia set $J$ of the associated polynomial semigroup $G$ and that $J$ is a thin fractal set. Moreover, $T_{\infty}$ respects the surrounding order (see Definition 1.13). The function $T_{\infty}$ is a complex analogue of the devil's staircase or Lebesgue's singular functions. For the detail of these results, see the second author's works 32, 28, 34.

As is well known, the iteration of polynomial maps $f_{c}(z)=z^{2}+c$ for $c$ in the Mandelbrot set (where the orbit of the sole critical point $\left\{f_{c}^{n}(0)\right\}$ is bounded in $\mathbb{C}$ ) provides a rich class of maps with many interesting properties. Many of these dynamic and structural properties are direct consequences of the boundedness of the critical orbit. It is then natural to look at the more general situation of polynomial semigroups with a bounded postcritical set. We discuss the dynamics of such polynomial semigroups as well as the structure of their Julia sets. For some properties of polynomial semigroups with a bounded finite postcritical set see also [26, 27, 29, 30, 31, 25, 33. This paper is a continuation of the program initiated in [29, 30, 31, 33. One may also see the paper [19, which is based on conference talks by the authors, where preliminary (weaker) versions of a portion of the results contained herein were announced and proven.

Definition 1.1. Let $G$ be a rational semigroup. We set

$$
F(G)=\{z \in \overline{\mathbb{C}} \mid G \text { is normal in a neighborhood of } z\} \text { and } J(G)=\overline{\mathbb{C}} \backslash F(G) .
$$

We call $F(G)$ the Fatou set of $G$ and $J(G)$ the Julia set of $G$. The Fatou set and Julia set of the semigroup generated by a single map $g$ is denoted by $F(g)$ and $J(g)$, respectively.

We quote the following results from [11. The Fatou set $F(G)$ is forward invariant under each element of $G$, i.e., $g(F(G)) \subset F(G)$ for all $g \in G$, and thus $J(G)$ is backward invariant under each element of $G$, i.e., $g^{-1}(J(G)) \subset J(G)$ for all $g \in G$. Furthermore, when the cardinality \#J(G) is three or more, $J(G)$ is the smallest closed subset of $\overline{\mathbb{C}}$ which contains three or more points and is backward invariant. Letting the backward orbit of $z$ be denoted by

$$
G^{-1}(z)=\bigcup_{g \in G} g^{-1}(\{z\}),
$$

we have that $J(G)=\overline{G^{-1}(z)}$ for any $z \in J(G)$ whose backward orbit contains three or more points. 
We should take a moment to note that the sets $F(G)$ and $J(G)$ are, however, not necessarily completely invariant under the elements of $G$. This is in contrast to the case of iteration dynamics, i.e., the dynamics of semigroups generated by a single rational function. For a treatment of alternatively defined completely invariant Julia sets of rational semigroups the reader is referred to [13, 14, 15, 18,

Although the Julia set of a rational semigroup $G$ may not be completely invariant, $J(G)$ has an interesting property. Namely, if $G$ is generated by a compact family $\left\{h_{\lambda}: \lambda \in \Lambda\right\}$ of rational maps, then $J(G)=\bigcup_{\lambda \in \Lambda} h_{\lambda}^{-1}(J(G))$. This property is called backward self-similarity. In particular, if $G=\left\langle h_{1}, \ldots, h_{m}\right\rangle$, then $J(G)=$ $\bigcup_{j=1}^{m} h_{j}^{-1}(J(G))$. From this property, the dynamics of rational semigroups can be regarded as "backward iterated function systems", and in the study of rational semigroups, we sometimes borrow and further develop techniques from iterated function systems and fractal geometry. For these things, see the second author's works [20] - 33] and 36, 37.

Note that $J(G)$ contains the Julia set of each element of $G$. Moreover, the following critically important result first due to Hinkkanen and Martin holds (see also [16]).

Theorem 1.2 ([1], Corollary 3.1). For rational semigroups $G$ with $\sharp(J(G)) \geq 3$, we have

$$
J(G)=\overline{\bigcup_{f \in G} J(f)} .
$$

Remark 1.3. Theorem 1.2 can be used to easily show that $F\left(\left\langle h_{\lambda}: \lambda \in \Lambda\right\rangle\right)$ is precisely the set of $z \in \overline{\mathbb{C}}$ which has a neighborhood on which every composition sequence generated by $\left\{h_{\lambda}: \lambda \in \Lambda\right\}$ is normal, i.e., has stable dynamics (see [39]).

In what follows we employ the following notation. The forward orbit of $z$ is given by $G(z)=\bigcup_{g \in G} g(\{z\})$. For any subset $A$ of $\overline{\mathbb{C}}$, we set $G^{-1}(A)=$ $\bigcup_{g \in G} g^{-1}(A)$. For any polynomial $g$, we denote the filled-in Julia set of $g$ by $K(g):=\left\{z \in \mathbb{C} \mid \bigcup_{n \in \mathbb{N}} g^{n}(\{z\})\right.$ is bounded in $\left.\mathbb{C}\right\}$. We note that for a polynomial $g$ with $\operatorname{deg}(g) \geq 2, J(g)=\partial K(g)$ and $K(g)$ is the polynomial hull of $J(g)$. The appropriate extension (to our situation with polynomial semigroups) of the concept of the filled-in Julia set is as follows. (See 11, 3, for other kinds of filled-in Julia sets.)

Definition 1.4. For a polynomial semigroup $G$, we set

$$
\hat{K}(G):=\{z \in \mathbb{C} \mid G(z) \text { is bounded in } \mathbb{C}\}
$$

and call $\hat{K}(G)$ the smallest filled-in Julia set.

Remark 1.5. We note that for all $g \in G$, we have $\hat{K}(G) \subset K(g)$ and $g(\hat{K}(G)) \subset$ $\hat{K}(G)$.

Definition 1.6. The postcritical set of a rational semigroup $G$ is defined by

$$
P(G)=\overline{\bigcup_{g \in G}\{\text { all critical values of } g: \overline{\mathbb{C}} \rightarrow \overline{\mathbb{C}}\}}(\subset \overline{\mathbb{C}}) .
$$

We say that $G$ is hyperbolic if $P(G) \subset F(G)$, and we say that $G$ is subhy-

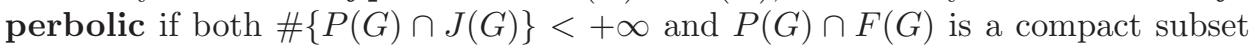
of $F(G)$. For research on (semi)hyperbolicity and the Hausdorff dimension of Julia sets of rational semigroups see [20, 21, 22, 23, 24, 29, 30, 31, 36, 33. 
Remark 1.7. It is clear that if the rational semigroup $G$ is hyperbolic, then each $g \in G$ is hyperbolic. However, the converse is not true. See Remark 5.2.

Definition 1.8. The planar postcritical set (or the finite postcritical set) of a polynomial semigroup $G$ is defined by

$$
P^{*}(G)=P(G) \backslash\{\infty\} .
$$

We say that a polynomial semigroup $G$ is postcritically bounded if $P^{*}(G)$ is bounded in $\mathbb{C}$.

Definition 1.9. Let $\mathcal{G}$ be the set of all polynomial semigroups $G$ with the following properties:

- each element of $G$ is of degree at least two and

- $P^{*}(G)$ is bounded in $\mathbb{C}$, i.e., $G$ is postcritically bounded.

Furthermore, we set $\mathcal{G}_{\text {con }}=\{G \in \mathcal{G} \mid J(G)$ is connected $\}$ and $\mathcal{G}_{\text {dis }}=\{G \in \mathcal{G} \mid$ $J(G)$ is disconnected $\}$.

Remark 1.10. If $G=\left\langle h_{\lambda}: \lambda \in \Lambda\right\rangle$, then

$$
P(G)=\overline{\bigcup_{\lambda \in \Lambda} \bigcup_{z \in C V\left(h_{\lambda}\right)}(G(z) \cup\{z\})},
$$

where $C V(h)$ denotes the critical values of $h$. From this one may, in the finitely generated case, use a computer to see if $G \in \mathcal{G}$, much in the same way as one verifies the boundedness of the critical orbit for the maps $f_{c}(z)=z^{2}+c$. The freely available software [17 can be used for this purpose.

Remark 1.11. Since $P(G)$ is forward invariant under $G$, we see that $G \in \mathcal{G}$ implies $P^{*}(G) \subset \hat{K}(G)$, and thus $P^{*}(G) \subset K(g)$ for all $g \in G$.

Remark 1.12. For a polynomial $g$ of degree two or more, it is well known that $\langle g\rangle \in \mathcal{G}$ if and only if $J(g)$ is connected (see 2, Theorem 9.5.1). Hence, for any $g \in G \in \mathcal{G}$, we have that $J(g)$ is connected. We note, however, that the analogous result for polynomial semigroups does not hold, as there are many examples where $G \in \mathcal{G}$ but $J(G)$ is not connected (see [38, 29, 30, 31, 33]).

See also 25] for an analysis of the number of connected components of $J(G)$ involving the inverse limit of the spaces of connected components of the realizations of the nerves of finite coverings $\mathcal{U}$ of $J(G)$, where $\mathcal{U}$ consists of backward images of $J(G)$ under finite word maps in $G$. In fact, the number of connected components of the Julia set of a finitely generated rational semigroup is deeply related to a new kind of cohomology (called the "interaction cohomology"), which has been introduced by the second author of this paper. Using this cohomology, one can also investigate the number of connected components of the Fatou set of a finitely generated rational semigroup.

The aim of this paper is to investigate what can be said about the structure of the Julia sets and the dynamics of semigroups $G \in \mathcal{G}$. We begin by examining the structure of the Julia set and note that a natural order (that is, respected by the backward action of the maps in $G$ ) can be placed on the components of $J(G)$, which then leads to implications on the connectedness of Fatou components.

Notation. For a polynomial semigroup $G \in \mathcal{G}$, we denote by $\mathcal{J}=\mathcal{J}_{G}$ the set of all connected components of $J(G)$ which do not include $\infty$. 
Definition 1.13. We place a partial order on the space of all non-empty connected sets in $\mathbb{C}$ as follows. For connected sets $K_{1}$ and $K_{2}$ in $\mathbb{C}$, " $K_{1} \leq K_{2}$ " indicates that $K_{1}=K_{2}$ or $K_{1}$ is included in a bounded component of $\mathbb{C} \backslash K_{2}$. Also, " $K_{1}<K_{2}$ " indicates $K_{1} \leq K_{2}$ and $K_{1} \neq K_{2}$. We call $\leq$ the surrounding order and read $K_{1}<K_{2}$ as " $K_{1}$ is surrounded by $K_{2}$ ".

Convention. When a set $K_{1}$ is contained in the unbounded component of $\overline{\mathbb{C}} \backslash K_{2}$ we say that $K_{1}$ is "outside" $K_{2}$.

Theorem 1.14 (29, 33]). Let $G \in \mathcal{G}$ (possibly infinitely generated). Then:

(1) $(\mathcal{J}, \leq)$ is totally ordered.

(2) Each connected component of $F(G)$ is either simply or doubly connected.

(3) For any $g \in G$ and any connected component $J$ of $J(G)$, we have that $g^{-1}(J)$ is connected. Let $g^{*}(J)$ be the connected component of $J(G)$ containing $g^{-1}(J)$. If $J \in \mathcal{J}$, then $g^{*}(J) \in \mathcal{J}$. If $J_{1}, J_{2} \in \mathcal{J}$ and $J_{1} \leq J_{2}$, then both $g^{-1}\left(J_{1}\right) \leq g^{-1}\left(J_{2}\right)$ and $g^{*}\left(J_{1}\right) \leq g^{*}\left(J_{2}\right)$.

Remark 1.15. We note that under the hypothesis of the above theorem $J_{1}<J_{2}$ for $J_{1}, J_{2} \in \mathcal{J}$ does not necessarily imply $g^{*}\left(J_{1}\right)<g^{*}\left(J_{2}\right)$, but only that $g^{*}\left(J_{1}\right) \leq$ $g^{*}\left(J_{2}\right)$ as can be seen in Example 4.7. The Julia set of a $G \in \mathcal{G}_{\text {dis }}$ is shown in Figure 1.

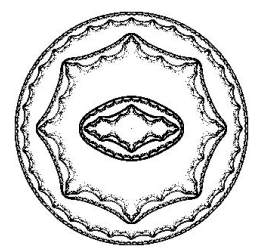

Figure 1 . The Julia set of $G=\left\langle h_{1}, h_{2}\right\rangle$, where $g_{1}(z):=z^{2}-1$, $g_{2}(z):=z^{2} / 4, h_{1}:=g_{1}^{2}, h_{2}:=g_{2}^{2} . G \in \mathcal{G}_{\text {dis }}$ and $G$ is hyperbolic.

We now present the main results of this paper, first giving some notation that will be needed to state our result on the existence of quasicircles in $J(G)$.

Notation. Given polynomials $\alpha_{1}$ and $\alpha_{2}$, we set $\Sigma_{2}=\left\{x=\left(\gamma_{1}, \gamma_{2}, \ldots\right): \gamma_{k} \in\right.$ $\left.\left\{\alpha_{1}, \alpha_{2}\right\}\right\}$. Then, for any $x=\left(\gamma_{1}, \gamma_{2}, \ldots\right) \in \Sigma_{2}$, we set $J_{x}$ equal to the set of points $z \in \overline{\mathbb{C}}$ where the sequence of functions $\left\{\gamma_{n} \circ \cdots \circ \gamma_{1}\right\}_{n \in \mathbb{N}}$ is not normal. This is sometimes called the Julia set along the trajectory (sequence) $x \in \Sigma_{2}$. See [21, 22, 24, 30, 31, 33] for much more on such fiberwise dynamics.

Theorem 1.16. Let $G \in \mathcal{G}_{\text {dis }}$ and let $A$ and $B$ be disjoint subsets of $\overline{\mathbb{C}}$. Suppose that $A$ is a doubly connected component of $F(G)$ and $B$ satisfies one of the following conditions:

- $B$ is a doubly connected component of $F(G)$,

- $B$ is the connected component of $F(G)$ with $\infty \in B$,

- $B=\hat{K}(G)$. 
Then $\partial A \cap \partial B=\emptyset$. Furthermore, $\bar{A}$ and $\bar{B}$ are separated by a Cantor family of quasicircles with uniform dilatation which all lie in $J(G)$. More precisely, there exist two elements $\alpha_{1}, \alpha_{2} \in G$ satisfying all of the following:

(1) There exists a non-empty open set $U$ in $\overline{\mathbb{C}}$ with $\alpha_{1}^{-1}(\bar{U}) \cap \alpha_{2}^{-1}(\bar{U})=\emptyset$ and $\alpha_{1}^{-1}(\bar{U}) \cup \alpha_{2}^{-1}(\bar{U}) \subset U$.

(2) $H=\left\langle\alpha_{1}, \alpha_{2}\right\rangle$ is hyperbolic.

(3) Letting $\Sigma_{2}$ denote the sequence space associated with $\left\{\alpha_{1}, \alpha_{2}\right\}$, we have

(a) $J(H)=\bigcup_{x \in \Sigma_{2}} J_{x}$ (disjoint union),

(b) for any component $J$ of $J(H)$ there exists a unique element $x \in \Sigma_{2}$ with $J=J_{x}$, and

(c) there exists a constant $K \geq 1$ such that any component $J$ of $J(H)$ is a $K$-quasicircle.

(4) $\left\{J_{x}\right\}_{x \in \Sigma_{2}}$ is totally ordered with $\leq$, consisting of mutually disjoint subsets of $J(H)$.

(5) For each $x \in \Sigma_{2}$, the set $J_{x}$ separates $\bar{A}$ from $\bar{B}$.

Remark 1.17. It should be noted that in the above theorem the quasicircles $J_{x}$ are all disjoint components of $J(H)$, but they may all lie in the same component of $J(G)$. See the proof of Theorem 1.23, where a semigroup is constructed such that there exist only a finite number of components of the Julia set.

Remark 1.18. There are many hyperbolic polynomial semigroups $G=\left\langle\alpha_{1}, \alpha_{2}\right\rangle \in$ $\mathcal{G}_{\text {dis }}$ such that for a generic $x \in \Sigma_{2}$, the fiberwise Julia set $J_{x}$ is a Jordan curve but not quasicircle, the unbounded component of $\overline{\mathbb{C}} \backslash J_{x}$ is a John domain, and the bounded component of $\overline{\mathbb{C}} \backslash J_{x}$ is not a John domain (see [31, 33, 30]). See Figure 1. This phenomenon does not occur in the usual iteration dynamics of a single polynomial.

Example 1.19. We give an example of a semigroup $G \in \mathcal{G}$ such that $J(G)$ is a "Cantor set of round circles". Let $f_{1}(z)=a z^{k}$ and $f_{2}(z)=b z^{j}$ for some positive integers $k, j \geq 2$. Then, for $|a|^{k-1} \neq|b|^{j-1}$, the sets $J\left(f_{1}\right)$ and $J\left(f_{2}\right)$ are disjoint circles centered at the origin. Let $A$ denote the closed annulus between $J\left(f_{1}\right)$ and $J\left(f_{2}\right)$. For positive integers $m_{1}$ and $m_{2}$ each greater than or equal to 2 (if $k$ and $j$ are not both equal to 2 , then $m_{1}=m_{2}=1$ will also suffice), we see that the iterates $g_{1}=f_{1}^{m_{1}}$ and $g_{2}=f_{2}^{m_{2}}$ will yield $A_{1}=g_{1}^{-1}(A) \cup g_{2}^{-1}(A) \subset A$, where $g_{1}^{-1}(A) \cap g_{2}^{-1}(A)=\emptyset$. Now iteratively define $A_{n+1}=g_{1}^{-1}\left(A_{n}\right) \cup g_{2}^{-1}\left(A_{n}\right)$ and note that for $G=\left\langle g_{1}, g_{2}\right\rangle$ we have $J(G)=\bigcap_{n=1}^{\infty} A_{n}$, since $J(G)$ is the smallest closed backward invariant (under each element of $G$ ) set which contains three or more points.

For our remaining results we need to note the existence of both a minimal element and a maximal element in $\mathcal{J}$ and state a few of their properties.

Theorem 1.20 (29, 33]). Let $G$ be a polynomial semigroup in $\mathcal{G}_{\text {dis }}$. Then there is a unique element $J_{\min }(G)$ (abbreviated by $\left.J_{\min }\right) \in \mathcal{J}$ such that $J_{\min }$ meets (and therefore contains) $\partial \hat{K}(G)$. Also, $\infty \in F(G)$ and there exists a unique element $J_{\max }(G)$ (abbreviated by $\left.J_{\max }\right) \in \mathcal{J}$ such that $J_{\max }$ meets (and therefore contains) $\partial U_{\infty}$, where $U_{\infty}$ is the simply connected component of $F(G)$ which contains $\infty$. 
Moreover, $\operatorname{int} \hat{K}(G) \neq \emptyset$. Furthermore, we have the following:

- $J_{\min } \leq J$ for all $J \in \mathcal{J}$,

- $J_{\max } \geq J$ for all $J \in \mathcal{J}$,

- $\hat{K}(G)$, and therefore $P^{*}(G)$, is contained in the polynomial hull of each $J \in \mathcal{J}$.

Remark 1.21. We see that $\partial \hat{K}(G) \subset J(G)$ when $G \in \mathcal{G}$, but, in general, we do not have $\partial \hat{K}(G)=J(G)$, unlike in iteration theory where $\partial K(g)=J(g)$ for polynomials $g$ of degree two or more. In fact, $\partial \hat{K}(G)$ might not even equal $J_{\min }(G)$ either (see Example 4.24).

Remark 1.22. When $G \in \mathcal{G}_{\text {con }}$ we will use the convention that $J_{\min }=J_{\max }=J(G)$ and note that it is still the case that $J_{\text {min }}$ meets $\partial \hat{K}$ and $P^{*}(G)$ is contained in the polynomial hull of each $J \in \mathcal{J}$. However, it is not necessarily the case that $\infty \in F(G)$, as exhibited by the example $\left\langle z^{2} / n: n \in \mathbb{N}\right\rangle$.

In the proofs of many results concerning postcritically bounded polynomial semigroups, it is critical to understand the distribution of the sets $J(g)$ where $g \in G$, especially when $g$ is a generator of $G$. In particular, it is important to understand the relationship between such $J(g)$ and the special components $J_{\min }$ and $J_{\max }$ of $J(G)$. In Section 4 we investigate such matters by carefully providing several results including Theorem 1.23 below.

In [29] it was shown that, for each positive integer $k$, there exists a semigroup $G \in$ $\mathcal{G}_{\text {dis }}$ with $2 k$ generators such that $J(G)$ has exactly $k$ components. Furthermore, in 25 it was shown that any semigroup in $\mathcal{G}$ generated by exactly three elements will have a Julia set with either one component or infinitely many components (examples where the number of components is one, $\aleph_{0}$ or uncountable were given). Hence we have the following question: For a fixed integer $k \geq 3$, what is the fewest number of generators that can produce a semigroup $G \in \mathcal{G}_{\text {dis }}$ with $\sharp \mathcal{J}=k$ ? The answer to this question is four, as stated in Theorem 1.23 below.

Theorem 1.23. For any $k \in \mathbb{N}$, there exists a 4-generator polynomial semigroup $H \in \mathcal{G}$ such that $\# \mathcal{J}_{H}=k$. Furthermore, $H$ can be chosen so that no $J \in$ $\mathcal{J}_{H} \backslash\left\{J_{\min }, J_{\max }\right\}$ meets the Julia set of any generator of $H$.

The next two results, whose proofs depend on understanding the distribution of the $J(g)$ within $J(G)$, concern the (semi)hyperbolicity of polynomial semigroups in $\mathcal{G}$. In particular, they show how one can build larger (semi)hyperbolic polynomial semigroups in $\mathcal{G}$ from smaller ones by including maps with certain properties. We first state two definitions.

Definition 1.24. We define Poly $=\{h: \overline{\mathbb{C}} \rightarrow \overline{\mathbb{C}} \mid h$ is a non-constant polynomial $\}$, endowed with the topology of uniform convergence on $\overline{\mathbb{C}}$ with respect to the spherical metric.

Remark 1.25. For later use we note that given an integer $d \geq 1$, a sequence $p_{n}$ of polynomials of degree $d$ converges to a polynomial $p$ in Poly if and only if the coefficients converge appropriately and $p$ is of degree $d$.

Definition 1.26. A rational semigroup $H$ is semihyperbolic if for each $z \in J(H)$ there exists a neighborhood $U$ of $z$ and a number $N \in \mathbb{N}$ such that for each $g \in H$ we have $\operatorname{deg}(g: V \rightarrow U) \leq N$ for each connected component $V$ of $g^{-1}(U)$. 
Theorem 1.27. Let $H \in \mathcal{G}, \Gamma$ be a compact family in Poly, and let $G=\langle H, \Gamma\rangle$ be the polynomial semigroup generated by $H$ and $\Gamma$. Suppose

(1) $G \in \mathcal{G}_{\text {dis }}$,

(2) $J(\gamma) \cap J_{\min }(G)=\emptyset$ for each $\gamma \in \Gamma$, and

(3) $H$ is semihyperbolic.

Then, $G$ is semihyperbolic.

Remark 1.28. Theorem 1.27 would not hold if we were to replace both instances of the word semihyperbolic with the word hyperbolic (see Example 5.1). However, with an additional hypothesis we do get the following result.

Theorem 1.29. Let $H \in \mathcal{G}, \Gamma$ be a compact family in Poly, and let $G=\langle H, \Gamma\rangle$ be the polynomial semigroup generated by $H$ and $\Gamma$. Suppose

(1) $G \in \mathcal{G}_{\text {dis }}$,

(2) $J(\gamma) \cap J_{\min }(G)=\emptyset$ for each $\gamma \in \Gamma$,

(3) $H$ is hyperbolic, and

(4) for each $\gamma \in \Gamma$, the critical values of $\gamma$ do not meet $J_{\min }(G)$. Then, $G$ is hyperbolic.

Remark 1.30. We note that hypothesis (3) can be replaced by the slightly weaker hypothesis that $P^{*}(H) \cap J(H)=\emptyset$ since if $\infty \in J(H) \subset J(G)$, then $J(G)$ is connected by Theorem 1.20, and so hypothesis (1) fails to hold. A similar remark can be made about hypothesis (3) in Theorem 1.27.

Remark 1.31. Theorems 1.27 and 1.29 do not require that $H$ or $G$ be generated by a finite, or even compact, subset of Poly.

The rest of this paper is organized as follows. In Section 2 we give the necessary background and tools required. In Section 3 we give the proof of Theorem 1.16 In Section 4 we provide a more detailed look at the distribution of $J(g)$ within $J(G)$, in particular, proving Theorem 1.23. In Section 5 we give the proofs of Theorems 1.27 and 1.29 along with Example 5.1 .

\section{BACKGROUND AND TOOLS}

We first state some notation to be used later.

Notation. Given any set $A \subset \overline{\mathbb{C}}$ we denote by $\bar{A}$ the closure of $A$ in $\overline{\mathbb{C}}$. For $z_{0} \in \mathbb{C}$ and $r, R>0$ we set $B\left(z_{0}, r\right)=\left\{z \in \mathbb{C}:\left|z-z_{0}\right|<r\right\}, C\left(z_{0}, r\right)=\left\{z \in \mathbb{C}:\left|z-z_{0}\right|=r\right\}$, and $\operatorname{Ann}\left(z_{0} ; r, R\right)=\left\{z \in \mathbb{C}: r<\left|z-z_{0}\right|<R\right\}$. Furthermore, given any set $C \subset \mathbb{C}$ we denote the $\epsilon$-neighborhood of $C$ by $B(C, \epsilon)=\bigcup_{z \in C} B(z, \epsilon)$.

Most often our understanding of the surrounding order $\leq$ given in Definition 1.13 will be applied to compact connected sets in $\mathbb{C}$, and so in this section we state many results which we will need later. Although not all connected compact sets in $\mathbb{C}$ are comparable in the surrounding order, we do have the following two lemmas whose proofs we leave to the reader.

Lemma 2.1. Given two connected compact sets $A$ and $B$ in $\mathbb{C}$ we must have exactly one of the following:

(1) $A<B$,

(2) $B<A$,

(3) $A \cap B \neq \emptyset$, 
(4) $A$ and $B$ are outside of each other, i.e., $A$ is a subset of the unbounded component of $\mathbb{C} \backslash B$ and $B$ is a subset of the unbounded component of $\mathbb{C} \backslash A$.

Definition 2.2. For a compact set $A \subset \mathbb{C}$ we define the polynomial hull $P H(A)$ of $A$ to be the union of $A$ and all bounded components of $\mathbb{C} \backslash A$.

Lemma 2.3. Let $A$ and $B$ be compact connected subsets of $\mathbb{C}$ such that $P H(A) \cap$ $P H(B) \neq \emptyset$. Then exactly one of the following holds:

(1) $A<B$,

(2) $B<A$,

(3) $A \cap B \neq \emptyset$.

Remark 2.4. We note that for compact connected sets $A$ and $B$ in $\mathbb{C}$, it follows that $A<B$ if and only if $P H(A)<B$, since the set $P H(A)$ is also compact and connected.

Lemma 2.5. Let $g$ be a polynomial of degree at least one and suppose $B \subset P H(A)$, where $g(B) \subset B$ and $A \subset \mathbb{C}$ is compact. Then $B \subset P H\left(g^{-1}(A)\right)$. In particular, if $g \in G \in \mathcal{G}$ and $P^{*}(G) \subset P H(A)$ where $A \subset \mathbb{C}$ is compact, then $P^{*}(G) \subset$ $P H\left(g^{-1}(A)\right)$.

Proof. Suppose $B \nsubseteq P H\left(g^{-1}(A)\right)$. Thus there exists $z_{0} \in B$ in the unbounded component $U$ of $\overline{\mathbb{C}} \backslash g^{-1}(A)$. Let $\gamma$ be a curve in $U$ connecting $z_{0}$ to $\infty$. Then $\Gamma=g \circ \gamma$ is a curve in $\overline{\mathbb{C}} \backslash A$ which connects $g\left(z_{0}\right)$ to $\infty$, which shows that $g\left(z_{0}\right) \notin$ $P H(A)$. Since $B$ is forward invariant we have that $g\left(z_{0}\right) \in B \backslash P H(A)$, which contradicts our hypothesis.

Corollary 2.6. Let $f, g \in G \in \mathcal{G}$. If $A$ is of the form $J \in \mathcal{J}, J(f), g^{-1}(J)$, or $g^{-1}(J(f))$, then $P^{*}(G) \subset P H(A)$.

Proof. By Theorem 1.20 we have $P^{*}(G) \subset P H(J)$ for all $J \in \mathcal{J}$. By Remark 1.11 $P^{*}(G) \subset K(f)=P H(J(f))$. The other cases then follow from Lemma 2.5

Lemma 2.7 (12]). Let $X$ be a compact metric space and let $f: X \rightarrow X$ be a continuous open map. Let $K$ be a compact connected subset of $X$. Then for each connected component $B$ of $f^{-1}(K)$, we have $f(B)=K$.

Lemma 2.8. Let $g$ be a polynomial with $d=\operatorname{deg}(g) \geq 1$ and let $K \subset \mathbb{C}$ be a connected compact set such that the unbounded component $U$ of $\overline{\mathbb{C}} \backslash K$ contains no critical values of $g$ other than $\infty$, i.e., the finite critical values of $g$ lie in $P H(K)$. Then $g^{-1}(K)$ is connected. Further, if $K_{1}$ is a connected compact set such that $K<K_{1}$, then $g^{-1}(K)<g^{-1}\left(K_{1}\right)$.

Proof. Set $V=g^{-1}(U)$ and note that $V$ contains no finite critical points of $g$. Thus by the Riemann-Hurwitz relation we have $\chi(V)+\delta_{g}(V)=d \chi(U)$, where $\chi(\cdot)$ denotes the Euler characteristic and $\delta_{g}(\cdot)$ is the deficiency. Since the hypotheses on $U$ imply $\delta_{g}(V)=d-1$ and $\chi(U)=1$, we see that $\chi(V)=1$. Hence the open and connected set $V$ is simply connected.

Suppose that $g^{-1}(K)$ is not connected. Then there exists a bounded component $V_{0}$ of $\mathbb{C} \backslash g^{-1}(K)$ which is not simply connected (see [2], Proposition 5.1.5). Thus there exists a Jordan curve $\gamma \subset V_{0}$ such that the bounded component $B$ of $\mathbb{C} \backslash \gamma$ contains some component $E$ of $g^{-1}(K)$. Hence $V_{0} \cup B$ is open and does not meet $V$. By Lemma 2.7 we have $g(E)=K$. Hence $g\left(V_{0} \cup B\right) \supset K \supset \partial U$, which, by 
the Open Mapping Theorem, implies $g\left(V_{0} \cup B\right)$ meets $U$, and thus $V_{0} \cup B$ meets $V$. This contradiction implies that $V_{0}$ is simply connected, and hence $g^{-1}(K)$ is connected.

Now suppose $K<K_{1}$. Let $U_{0}$ be the bounded component of $\mathbb{C} \backslash K_{1}$ such that $U_{0} \supset K$ and let $U_{1}$ be the unbounded component of $\mathbb{C} \backslash K_{1}$. Hence $g^{-1}\left(U_{0}\right)$ does not meet $V_{1}=g^{-1}\left(U_{1}\right)$. Hence $P H\left(g^{-1}\left(K_{1}\right)\right)=\mathbb{C} \backslash V_{1} \supset g^{-1}\left(U_{0}\right) \supset g^{-1}(K)$. Since $g^{-1}\left(K_{1}\right) \cap g^{-1}(K)=\emptyset$ (which follows from the fact that $K_{1} \cap K=\emptyset$ ), we conclude that $g^{-1}\left(K_{1}\right)>g^{-1}(K)$.

Corollary 2.9. Let $g, h \in G \in \mathcal{G}$ and $J \in \mathcal{J}$. Then $g^{-1}(J)$ and $g^{-1}(J(h))$ are connected. Furthermore, $J_{1}<J_{2}$ for $J_{1}, J_{2} \in \mathcal{J}$ implies $g^{-1}\left(J_{1}\right)<g^{-1}\left(J_{2}\right)$, and $J\left(h_{1}\right)<J\left(h_{2}\right)$ for $h_{1}, h_{2} \in G$ implies $g^{-1}\left(J\left(h_{1}\right)\right)<g^{-1}\left(J\left(h_{2}\right)\right)$.

Proof. Any finite critical value of $g$ must lie in $P^{*}(G) \subset P H(J) \cap P H(J(h))$ by Corollary 2.6. The result then follows immediately from Lemma 2.8.

Corollary 2.10. Let $f, g \in G \in \mathcal{G}$. For any two sets $A$ and $B$ of the form $J \in \mathcal{J}, J(f), g^{-1}(J)$, or $g^{-1}(J(f))$, exactly one of the following must hold:

(1) $A<B$,

(2) $B<A$,

(3) $A \cap B \neq \emptyset$.

Proof. This is immediate from Corollary 2.9. Corollary 2.6 and Lemma 2.3.

The following lemma will allow one to understand the surrounding order through an imbedding, of sorts, into the real numbers.

Lemma 2.11. Suppose $z_{0} \in P H\left(C_{1}\right) \cap P H\left(C_{2}\right)$, where $C_{1}$ and $C_{2}$ are disjoint compact connected sets in $\mathbb{C}$. Then $\operatorname{dist}\left(z_{0}, C_{1}\right)<\operatorname{dist}\left(z_{0}, C_{2}\right)$ if and only if $C_{1}<$ $\mathrm{C}_{2}$ in the surrounding order.

Proof. First suppose that $d_{2}=\operatorname{dist}\left(z_{0}, C_{2}\right)>\operatorname{dist}\left(z_{0}, C_{1}\right)$. Then we have $B\left(z_{0}, d_{2}\right)$ $\subset P H\left(C_{2}\right)$. Since $B\left(z_{0}, d_{2}\right) \cap C_{1} \neq 0$ and $C_{1} \cap C_{2}=\emptyset$, we must have that the bounded component of $\overline{\mathbb{C}} \backslash C_{2}$ which contains the connected set $B\left(z_{0}, d_{2}\right)$ also contains the connected set $C_{1}$. Thus $C_{2}>C_{1}$.

Suppose $C_{1}<C_{2}$. Letting $d_{1}=\operatorname{dist}\left(z_{0}, C_{1}\right)$ we see that $\overline{B\left(z_{0}, d_{1}\right)} \subset \operatorname{PH}\left(C_{1}\right)<$ $C_{2}$ implies that $\overline{B\left(z_{0}, d_{1}\right)}$ must not meet $C_{2}$, i.e., $d_{1}<\operatorname{dist}\left(z_{0}, C_{2}\right)$.

Lemma 2.12. Let $\left\{C_{\alpha}\right\}_{\alpha \in \mathcal{A}}$ be a collection of non-empty compact connected sets in $\mathbb{C}$ that are linearly ordered by the surrounding order $\leq$. Suppose $\left\{C_{\beta}\right\}_{\beta \in \mathcal{B}}$ is a subcollection of $\left\{C_{\alpha}\right\}_{\alpha \in \mathcal{A}}$ such that $\overline{\bigcup_{\beta \in \mathcal{B}} C_{\beta}} \subset \bigcup_{\alpha \in \mathcal{A}} C_{\alpha}$. Then both inf $\operatorname{in}_{\beta \in \mathcal{B}} C_{\beta}$ and $\sup _{\beta \in \mathcal{B}} C_{\beta}$ exist and are in $\left\{C_{\alpha}\right\}_{\alpha \in \mathcal{A}}$.

Proof. By compactness and the linear ordering on $\left\{C_{\alpha}\right\}_{\alpha \in \mathcal{A}}$, one can quickly show that the collection $\left\{P H\left(C_{\alpha}\right)\right\}_{\alpha \in \mathcal{A}}$ satisfies the finite intersection property. Thus there exists some $z_{0} \in \bigcap_{\alpha \in \mathcal{A}} P H\left(C_{\alpha}\right)$. For each $\beta \in \mathcal{B}$, let $r_{\beta}=\operatorname{dist}\left(z_{0}, C_{\beta}\right)$ and consider $r_{0}=\inf r_{\beta}$.

We only need to consider the case where $r_{0}<r_{\beta}$ for all $\beta \in \mathcal{B}$, since if $r_{\beta_{0}}=r_{0}$, then clearly, by Lemma 2.11, $C_{\beta_{0}}=\inf _{\beta \in \mathcal{B}} C_{\beta}$. Select a strictly decreasing sequence $r_{\beta_{n}} \rightarrow r_{0}$. By Lemma 2.11 we have that $C_{\beta_{1}}>C_{\beta_{2}}>\ldots$ Let $z_{\beta_{n}} \in C_{\beta_{n}}$ be arbitrary. Without loss of generality we may assume that $z_{\beta_{n}} \rightarrow a_{0} \in \mathbb{C}$. By hypothesis there exists $C_{\alpha_{0}}$ which contains $a_{0}$. We will now show that $C_{\alpha_{0}}=$ $\inf _{\beta \in \mathcal{B}} C_{\beta}$. 
Fixing $\beta \in \mathcal{B}$ and applying Lemma 2.11 we see that $\left\{a_{0}\right\}<C_{\beta}$, since the sequence $\left(z_{\beta_{k}}\right)_{k \geq n}$ must lie in $P H\left(C_{\beta_{n}}\right)<C_{\beta}$ for large $n$ (whenever $r_{\beta_{n}}<r_{\beta}$ ). Thus we must have $C_{\alpha_{0}}<C_{\beta}$ for all $\beta \in \mathcal{B}$. Hence $C_{\alpha_{0}}$ is a lower bound for $\left\{C_{\beta}\right\}_{\beta \in \mathcal{B}}$. Suppose that $C_{\alpha_{1}}>C_{\alpha_{0}}$. It must then be the case that $\left\{a_{0}\right\}<C_{\alpha_{1}}$, and so it follows that $\left\{z_{\beta_{n}}\right\}<C_{\alpha_{1}}$ for large $n$. Thus $C_{\beta_{n}}<C_{\alpha_{1}}$ for large $n$, implying that $C_{\alpha_{1}}$ is not a lower bound for $\left\{C_{\beta}\right\}_{\beta \in \mathcal{B}}$. We conclude that $C_{\alpha_{0}}=\inf _{\beta \in \mathcal{B}} C_{\beta}$.

The proof that $\sup _{\beta \in \mathcal{B}} C_{\beta}$ exists in $\left\{C_{\alpha}\right\}_{\alpha \in \mathcal{A}}$ follows a similar argument using $\sup r_{\beta}$ and Lemma 2.11. We omit the details.

By the proof of the above lemma we see that if $\overline{\bigcup_{\beta \in \mathcal{B}} C_{\beta}}=\bigcup_{\beta \in \mathcal{B}} C_{\beta}$, then both $\inf _{\beta \in \mathcal{B}} C_{\beta}$ and $\sup _{\beta \in \mathcal{B}} C_{\beta}$ are in $\left\{C_{\beta}\right\}_{\beta \in \mathcal{B}}$. Thus we have the following.

Lemma 2.13. Let $\left\{C_{\beta}\right\}_{\beta \in \mathcal{B}}$ be a collection of compact connected sets in $\mathbb{C}$ that are linearly ordered by the surrounding order $\leq$. If $\overline{\bigcup_{\beta \in \mathcal{B}} C_{\beta}}=\bigcup_{\beta \in \mathcal{B}} C_{\beta}$, then we can conclude that both $\min _{\beta \in \mathcal{B}} C_{\beta}$ and $\max _{\beta \in \mathcal{B}} C_{\beta}$ exist.

Lemma 2.14. Let $f \in G \in \mathcal{G}$. Let $K$ be a connected compact set in $\mathbb{C}$ such that $P H(K) \supset P^{*}(f)$.

(a) Let $J(f)>K$. Then $J(f)>f^{-1}(K)$. Also, $f^{-1}(K)>K$ or $f^{-1}(K) \cap K \neq \emptyset$. $\emptyset$.

(b) Let $J(f)<K$. Then $J(f)<f^{-1}(K)$. Also, $f^{-1}(K)<K$ or $f^{-1}(K) \cap K \neq$

Proof. We now prove (a). We first note that $J(f)=f^{-1}(J(f))>f^{-1}(K)$ follows immediately from Lemma 2.8. Since $P^{*}(f) \subset P H(K)<J(f)$ we see that $f$ cannot have a Siegel disk or parabolic fixed point. Hence, $f$ must have a finite attracting fixed point $z_{0}$. Furthermore, since $P H(K)$ is connected and $P^{*}(f) \subset P H(K)<$ $J(f)$, it is clear that there can be only one attracting fixed point for $f$ and $K$ must lie in the immediate attracting basin $A_{f}\left(z_{0}\right)$. Since $P H\left(f^{-1}(K)\right) \supset P^{*}(f)$ by Lemma 2.5 we see that $f^{-1}(K)$ also lies in $A_{f}\left(z_{0}\right)$. Hence $A_{f}\left(z_{0}\right)$ must be completely invariant under $f$. This implies $F(f)$ has only two components, $A_{f}(\infty)$ and $A_{f}\left(z_{0}\right)$, each of which is simply connected (see [2], Theorem 5.6.1).

Letting $\varphi: A_{f}\left(z_{0}\right) \rightarrow B(0,1)$ be the Riemann map such that $z_{0} \mapsto 0$, then one may apply Schwarz's Lemma to the degree greater than or equal to two (finite Blaschke product) map $B=\varphi \circ f \circ \varphi^{-1}$ to show that any point mapped to a point of maximum modulus of $\varphi(K)$ must lie outside of $\varphi(K)$. Thus either $f^{-1}(K)>K$ or $f^{-1}(K) \cap K \neq \emptyset$.

Part (b) is proved more easily than (a) since it is already known that $A_{f}(\infty)$ is simply connected. Then one can similarly examine the Riemann map from $A_{f}(\infty)$ to $B(0,1)$ such that $\infty \mapsto 0$.

We note that Theorem 1.20 along with the proof of part (a) above, with $K=$ $J_{\min }$, proves the following (which has been already shown in [29, 33]).

Lemma 2.15. Let $f \in G \in \mathcal{G}$ be such that $J(f)>J_{\min }$, i.e., $J(f) \cap J_{\min }=\emptyset$. Then $f$ has an attracting fixed point $z_{0} \in \mathbb{C}$ and $F(f)$ consists of just two simply connected immediate attracting basins $A_{f}(\infty)$ and $A_{f}\left(z_{0}\right)$.

We note that the maps $f=h_{1}^{2}$ and $g=h_{2}^{2}$, where $h_{1}(z)=z^{2}-1$ and $h_{2}(z)=$ $z^{2} / 4$, generate $G=\langle f, g\rangle \in \mathcal{G}_{\text {dis }}$, where $f$ has two finite attracting fixed points (see [31, 33]). Thus we see that the condition $J(f) \cap J_{\min }=\emptyset$ in the above lemma is indeed necessary. We also note that for this $G$, the phenomena in Remark 1.18 holds. See Figure 1 
Lemma 2.16. Let $f \in G \in \mathcal{G}$. Let $K$ be a connected set in $J(G)$ containing three or more points such that $f^{-n}(K)$ is also connected for each $n \in \mathbb{N}$. If $f^{-1}(K) \cap K \neq \emptyset$, then $J(f)$ and $K$ are contained in the same component $J \in \mathcal{J}$.

Proof. The lemma follows from the fact that the connected set $\overline{\bigcup_{n=0}^{\infty} f^{-n}(K)}$ in $J(G)$ must meet $J(f)$.

We now present a general topological lemma that will be used to justify a corollary which will be needed later.

Lemma 2.17. Let $\left\{C_{\alpha}\right\}_{\alpha \in \mathcal{A}}$ be a collection of compact connected sets in $\overline{\mathbb{C}}$. Let $\epsilon>0$ and let $C$ be any connected component of $\overline{\bigcup_{\alpha \in \mathcal{A}} C_{\alpha}}$. Then there exists $\alpha \in \mathcal{A}$ such that $C_{\alpha} \subset B(C, \epsilon)$.

Proof. Choose any $z \in C$ and let $\alpha_{n} \in \mathcal{A}$ be such that $\operatorname{dist}\left(z, C_{\alpha_{n}}\right) \rightarrow 0$. By compactness in the topology generated by the Hausdorff metric on the space of non-empty compact subsets of $\overline{\mathbb{C}}$, we then may conclude (by passing to subsequence if necessary) that $C_{\alpha_{n}} \rightarrow K$ for some non-empty connected compact set $K$, which therefore must contain $z$ and hence be contained in $C$. Thus for large $n$ we have $B(C, \epsilon) \supset B(K, \epsilon) \supset C_{\alpha_{n}}$.

Using the fact that $J(g)$ is connected whenever $g \in G \in \mathcal{G}$, we clearly obtain the following slight generalization of Lemma 4.2 in [29].

Corollary 2.18. Let $\left\{g_{\lambda}\right\}_{\lambda \in \Lambda} \subset G \in \mathcal{G}$. Let $\epsilon>0$ and let $C$ be any connected component of $\overline{\bigcup_{\lambda \in \Lambda} J\left(g_{\lambda}\right)}$. Then there exists $\lambda \in \Lambda$ such that $J\left(g_{\lambda}\right) \subset B(C, \epsilon)$.

In particular, we apply Theorem 1.2 to obtain that if $\left\{g_{\lambda}\right\}_{\lambda \in \Lambda}=G \in \mathcal{G}$ and $J \in \mathcal{J}$, then for every $\epsilon>0$ there exists $g \in G$ such that $J(g) \subset B(J, \epsilon)$.

\section{Proof of Theorem 1.16}

We first present a definition and a lemma that will assist in the proof of Theorem 1.16 ,

Definition 3.1. For compact connected sets $K_{1}$ and $K_{2}$ in $\mathbb{C}$ such that $K_{1}<K_{2}$ we define $\operatorname{Ann}\left(K_{1}, K_{2}\right)=U \backslash P H\left(K_{1}\right)$, where $U$ is the bounded component of $\mathbb{C} \backslash K_{2}$ which contains $K_{1}$. Thus $\operatorname{Ann}\left(K_{1}, K_{2}\right)$ is the open doubly connected region "between" $K_{1}$ and $K_{2}$.

Remark 3.2. For any compact connected set $A \subset A n n\left(K_{1}, K_{2}\right)$ we immediately see that $A<K_{2}$ and, by Lemma 2.1, either $K_{1}$ and $A$ are outside of each other or $K_{1}<A$.

Lemma 3.3. Let $f, g \in G \in \mathcal{G}$ be such that $J(f)$ and $J(g)$ lie in different components of $J(G)$ with $J(f)<J(g)$. Then for any fixed $n, m \in \mathbb{N}$ there exists $h, k \in G$ such that $f^{-(n+1)}(J(g))<J(h)<f^{-n}(J(g))$ and $g^{-m}(J(f))<J(k)<$ $g^{-(m+1)}(J(f))$.

Proof. Corollary 2.6. Lemma 2.14(a), and Lemma 2.16 show that $g^{-1}(J(f))>$ $J(f)$. Set $X=g^{-1}(J(f)), A=g^{-m}(J(f))$ and $B=g^{-(m+1)}(J(f))$, and note that $J(f)<A<B$ by Lemma 2.8 Keeping Lemma 2.15 in mind, we may choose $\ell \in \mathbb{N}$ large enough so that $f^{-\ell}(B) \subset A n n(J(f), X)$. Then $g^{-m}\left(f^{-\ell}(\overline{A n n(A, B)})\right) \subset$ $g^{-m}(A n n(J(f), X)) \subset A n n(A, B) \subset \overline{A n n(A, B)}$, which implies that $k=f^{\ell} \circ g^{m} \in$ 
$G$ is such that $J(k) \subset \operatorname{Ann}(A, B)$. Since by Corollary 2.10 we must have either $J(k)<A$ or $A<J(k)$, we see by construction that $A<J(k)$ must hold.

The other result is proved similarly.

We will require the following result which was proved via fiberwise quasiconformal surgery by the second author.

Proposition 3.4 (31, Proposition 2.25). Let $G=\left\langle\alpha_{1}, \alpha_{2}\right\rangle \in \mathcal{G}$ be hyperbolic such that $P^{*}(G)$ is contained in a single component of $\operatorname{int}(\hat{K}(G))$. Then there exists $K \geq 1$ such that for all sequences $x \in \Sigma_{2}$, the set $J_{x}$ is a $K$-quasicircle.

Remark 3.5. Under the hypotheses above we know that for each $g \in G$, the set $J(g)$ is a quasicircle (see [8, p. 102). But the above result shows much more, as it shows that the Julia sets along sequences are also all quasicircles and that all such quasicircles have uniform dilations.

We can now present the proof of Theorem 1.16.

Proof of Theorem 1.16. We first give a proof in the case that $A$ and $B$ are doubly connected components of $F(G)$. Since the doubly connected components of $F(G)$ are linearly ordered by $\leq$, we may assume without loss of generality that $A<B$.

Let $\gamma_{A}$ be a non-trivial curve in $A$ (i.e., $\gamma_{A}$ separates the components of $\mathbb{C} \backslash A$ ) and let $\gamma_{B}$ be a non-trivial curve in $B$. Since $J(G)=\overline{\bigcup_{g \in G} J(g)}$, the bounded component of $\mathbb{C} \backslash \gamma_{A}$ and $\operatorname{Ann}\left(\gamma_{A}, \gamma_{B}\right)$ both meet $J(G)$, and both $A$ and $B$ do not meet $J(G)$, there must exist maps $f, g \in G$ such that $J(f)<\gamma_{A}$ and $\gamma_{A}<$ $J(g)<\gamma_{B}$. Note then that $J(g)<B$ since $J(g) \cap B=\emptyset$. Since $J(f)$ and $J(g)$ lie in different components of $J(G)$ (separated by $A$ ), $J(g) \subset \overline{\bigcup_{n \in \mathbb{N}} g^{-n}(J(f))}$, and each $g^{-n}(J(f)) \cap A=\emptyset$, there exists $n_{0} \in \mathbb{N}$ such that $g^{-n_{0}}(J(f))>\gamma_{A}$ and thus $g^{-n_{0}}(J(f))>A$. By Lemma 3.3 there exists $k \in G$ such that $A<g^{-n_{0}}(J(f))<$ $J(k)<g^{-\left(n_{0}+1\right)}(J(f))<J(g)$.

We now find a subsemigroup $H^{\prime}$ that satisfies conclusions (1) - (4) of the theorem. Keeping Lemma 2.15 in mind, we see that we may choose $m_{1}, m_{2} \in \mathbb{N}$ large (as in Example 1.19), such that $\beta_{1}=k^{m_{1}}$ and $\beta_{2}=g^{m_{2}}$ generate a subsemigroup $H^{\prime}$ of $G$ where $J\left(H^{\prime}\right)$ is disconnected and contained in $\overline{A n n(J(k), J(g))}$. Further, $H^{\prime}$ is hyperbolic since $P^{*}\left(H^{\prime}\right) \subset P^{*}(G) \subset K(f)<J(k)$. By choosing $U$ to be a suitable open set containing $\overline{A n n(J(k), J(g))}$, we see that $H^{\prime}$ satisfies parts (1) and (2) of the theorem.

By Theorem 2.14(2) in 22], the hyperbolicity of $H^{\prime}$ implies $J\left(H^{\prime}\right)=\bigcup_{x \in \Sigma_{2}^{\prime}} J_{x}$, where $\Sigma_{2}^{\prime}$ is the sequence space corresponding to the maps $\beta_{1}$ and $\beta_{2}$. The fact that $J_{x_{1}} \neq J_{x_{2}}$ when $x_{1} \neq x_{2}$ follows in much the same way as the proof that the standard middle-third Cantor set is totally disconnected. We present the details now. First we define $\sigma$ to be the shift map on $\Sigma_{2}^{\prime}$ given by $\sigma\left(\gamma_{1}, \gamma_{2}, \ldots\right)=\left(\gamma_{2}, \gamma_{3}, \ldots\right)$. Then, for $x=\left(\gamma_{1}, \gamma_{2}, \ldots\right)$, one can show by using the definition of normality that $J_{x}=$ $\gamma_{1}^{-1}\left(J_{\sigma(x)}\right)$, and thus by induction

$$
J_{x}=\gamma_{1}^{-1}\left(J_{\sigma(x)}\right)=\cdots=\left(\gamma_{n} \circ \cdots \circ \gamma_{1}\right)^{-1}\left(J_{\sigma^{n}(x)}\right) \subset\left(\gamma_{n} \circ \cdots \circ \gamma_{1}\right)^{-1}\left(J\left(H^{\prime}\right)\right) .
$$

Thus $J_{x} \subset \bigcap_{n=1}^{\infty}\left(\gamma_{n} \circ \cdots \circ \gamma_{1}\right)^{-1}\left(J\left(H^{\prime}\right)\right)$. But by (induction on) condition (1) we can see that this intersection will produce distinct sets for distinct sequences in $\Sigma^{\prime}$. Thus we have shown that $J_{x_{1}} \neq J_{x_{2}}$ when $x_{1} \neq x_{2}$. 
Each $J_{x}$ is connected by Lemma 3.6 in 31. Hence we have shown parts 3(a) and 3(b). Now part (4) is then clear by 3(a), 3(b), and Theorem 1.14(1). Part 3(c) now follows directly from Proposition 3.4

We have thus shown that $H^{\prime}=\left\langle\beta_{1}, \beta_{2}\right\rangle$ satisfies items (1) - (4) of the theorem, but it is not certain that $J(g)$ does not meet $\bar{B}$, and so (5) remains in question. However, letting $\alpha_{1}=\beta_{1}$ and $\alpha_{2}=\beta_{1} \circ \beta_{2}$ (note $J\left(\alpha_{2}\right)<J\left(\beta_{2}\right)<B$ ), we see that $H=\left\langle\alpha_{1}, \alpha_{2}\right\rangle$ will satisfy (1) - (5). We have thus proved the result in the case that $A$ and $B$ are both doubly connected Fatou components.

Consider the case where $B$ is the unbounded component of $F(G)$ containing $\infty$. As above we obtain $f, g \in G$ such that $J(f)<\gamma_{A}<J(g)$, where $\gamma_{A}$ is a non-trivial curve in $A$. We then follow the above method to complete the proof.

Finally, we consider the case where $B=\hat{K}$. As above we obtain $f, g \in G$ such that $J(g)<\gamma_{A}<J(f)$, where $\gamma_{A}$ is a non-trivial curve in $A$. We then follow the above method, noting that the surrounding order inequalities are now reversed from above, to complete the proof.

\section{Structural properties of $\mathcal{J}$}

In this section we discuss issues related to the topological nature of $\mathcal{J}$ as well as issues related to the question of where the "small" Julia sets $J(g)$ for $g \in G$ reside inside of the larger Julia set $J(G)$. In particular, we investigate the question of when it is the case that a given $J \in \mathcal{J}$ must contain $J(g)$ for some $g \in G$. Since $J_{\min }$ and $J_{\max }$ play special roles we will be particularly interested in when these components of $J(G)$ have this property. When $G=\left\langle g_{\lambda}: \lambda \in \Lambda\right\rangle$, it is of particular interest to know which $J \in \mathcal{J}$ meet $J\left(g_{\lambda}\right)$ for some $\lambda \in \Lambda$. The first result in this direction is the following, which appears as Proposition 2.24 in [29].

Proposition 4.1. If $G \in \mathcal{G}$ is generated by a compact family in Poly, then both $J_{\min }$ and $J_{\max }$ must contain the Julia set of one of the generating maps of $G$.

In order to succinctly discuss such issues we make the following definitions.

Definition 4.2. Let $G=\left\langle h_{\lambda}: \lambda \in \Lambda\right\rangle \in \mathcal{G}$. We say that $J \in \mathcal{J}$ has property ( $\star$ ) if $J$ contains $J(g)$ for some $g \in G$. We say that $J \in \mathcal{J}$ has property $(\star \lambda)$ if $J$ contains $J\left(h_{\lambda}\right)$ for some generator $h_{\lambda} \in G$.

Remark 4.3. A given rational semigroup $G$ may have multiple generating sets. For example, the whole semigroup itself can always be taken as a generating set. However, in this paper when it is written that $G=\left\langle h_{\lambda}: \lambda \in \Lambda\right\rangle$, it is assumed that this generating set is fixed and thus the property $(\star \lambda)$ is always in relation to this given generating set.

Lemma 4.4. Let $G=\left\langle h_{\lambda}: \lambda \in \Lambda\right\rangle \in \mathcal{G}$.

a) If $J_{\min }$ has property $(\star)$, then $J_{\min }$ has property $(\star \lambda)$.

b) If $J_{\max }$ has property $(\star)$, then $J_{\max }$ has property $(\star \lambda)$.

Remark 4.5. Lemma 4.4 does not apply to a general $J \in \mathcal{J}$. Indeed, in Example 1.19 we see that only $J_{\min }$ and $J_{\max }$ have property $(\star \lambda)$, although infinitely many other $J \in \mathcal{J}$ have property $(\star)$.

Proof. Suppose $J(g) \subset J_{\min }$ for $g=h_{\lambda_{1}} \circ \cdots \circ h_{\lambda_{k}}$ and $J_{\min } \cap J\left(h_{\lambda}\right)=\emptyset$ for all $\lambda \in \Lambda$. By Corollary 2.10 we have $J_{\min }<J\left(h_{\lambda}\right)$ for all $\lambda \in \Lambda$, and thus by Lemma 2.14. 
Lemma 2.16, and Theorem 1.20, we have $J_{\min }<h_{\lambda}^{-1}\left(J_{\min }\right)$. So it also follows from Corollary 2.10 that $J(g)<h_{\lambda}^{-1}(J(g))$ for all $\lambda \in \Lambda$. Thus $J(g)<h_{\lambda_{1}}^{-1}(J(g))$, and by Lemma $2.8 J(g)<h_{\lambda_{2}}^{-1}(J(g))<h_{\lambda_{2}}^{-1} h_{\lambda_{1}}^{-1}(J(g))$. By repeated application of this argument we then get that $J(g)<h_{\lambda_{k}}^{-1}(J(g))<\cdots<h_{\lambda_{k}}^{-1} \cdots h_{\lambda_{1}}^{-1}(J(g))=$ $g^{-1}(J(g))=J(g)$, which is a contradiction. From this part (a) follows.

Part (b) follows in a similar manner.

Corollary 4.6. If $J_{\min }$ (respectively, $J_{\max }$ ) has non-empty interior, then $J_{\min }$ (respectively, $J_{\max }$ ) has property $(\star \lambda)$.

Proof. Suppose $\operatorname{int}\left(J_{\min }\right) \neq \emptyset$. Since $J(G)=\overline{\bigcup_{g \in G} J(g)}$ some $J(g)$ must meet $\operatorname{int}\left(J_{\min }\right)$. Thus it follows from Lemma 4.4 that $J_{\min }$ has property $(\star \lambda)$.

It is not always the case, however, that $J_{\min }$ and $J_{\max }$ have property $(\star \lambda)$.

Example 4.7. We will give an example of an infinitely generated $G \in \mathcal{G}_{\text {dis }}$ such that

(1) $J_{\max }$ does not have property $(\star \lambda)$,

(2) $\# \mathcal{J}=\aleph_{0}$, and

(3) there exists $J^{\prime}, J^{\prime \prime} \in \mathcal{J}$ and $g \in G$ such that $J^{\prime}<J^{\prime \prime}$ and $g^{*}\left(J^{\prime}\right)=g^{*}\left(J^{\prime \prime}\right)$.

Set $b_{n}=2-1 / n$ for $n \in \mathbb{N}$ and $\epsilon_{n}=\min \left\{\frac{b_{n+1}-b_{n}}{10}, \frac{b_{n}-b_{n-1}}{10}\right\}$. Set $A=\overline{\operatorname{Ann}(0 ; 1 / 2,2)}$, $A_{n}=\operatorname{Ann}\left(0 ; b_{n}-\epsilon_{n}, b_{n}+\epsilon_{n}\right)$ and $A_{n}^{\prime}=\operatorname{Ann}\left(\epsilon_{n} ; b_{n}-\epsilon_{n}, b_{n}+\epsilon_{n}\right)$. Note that the set $\tilde{A}_{n}:=\overline{A_{n} \cup A_{n}^{\prime}} \subset \overline{A n n\left(0 ; b_{n}-2 \epsilon_{n}, b_{n}+2 \epsilon_{n}\right)}$, and so, by the choice of the $\epsilon_{n}$, the $\tilde{A}_{n}$ are disjoint. Choose polynomials $f_{n}$ and $g_{n}$ such that $J\left(f_{n}\right)=C\left(0, b_{n}\right)$ and $J\left(g_{n}\right)=C\left(\epsilon_{n}, b_{n}\right)$. Choose $m_{n} \in \mathbb{N}$ large enough so that $h_{n}=f_{n}^{m_{n}}$ yields $h_{n}^{-1}(A) \subset$ $A_{n}, h_{n}(\overline{B(0,1 / 2)}) \subset B(0,1 / 2)$ and $h_{n}(\{|z| \geq 2\}) \subset\{|z|>5\}$. Choose $j_{n} \in \mathbb{N}$ large enough so that $k_{n}=g_{n}^{j_{n}}$ yields $k_{n}^{-1}(A) \subset A_{n}^{\prime}, k_{n}(\overline{B(0,1 / 2)}) \subset B(0,1 / 2)$ and

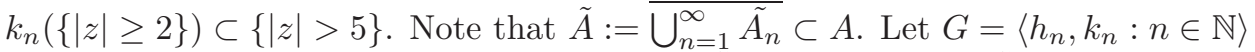
and note that $P^{*}(G) \subset B(0,1 / 2)$ and $G^{-1}(\tilde{A}) \subset G^{-1}(A) \subset \tilde{A}$, which implies $J(G) \subset \overline{G^{-1}(\tilde{A})} \subset \tilde{A}$.

We see by forward invariance that $\{|z|>2\} \subset F(G)$, but note that $C(0,2) \in \mathcal{J}$ (since the open sets $\operatorname{Ann}\left(\tilde{A}_{n}, \tilde{A}_{n+1}\right)$ are all in $\left.F(G)\right)$. Also, for no $g \in G$ does $J(g)$ meet $C(0,2)$. Otherwise, there would exist $z_{0} \in C(0,2) \cap J(g)$ such that $\left|g\left(z_{0}\right)\right| \leq 2$, contradicting the fact that each $g \in G$ maps $C(0,2)$ into $\{|z|>5\}$. Thus $J_{\max }=C(0,2)$ fails to have property $(\star)$.

We now show that $\# \mathcal{J}=\aleph_{0}$. Letting $J_{n} \in \mathcal{J}$ be such that $J_{n}$ contains the overlapping sets $J\left(h_{n}\right)$ and $J\left(k_{n}\right)$, we note that, since $J_{n} \subset \tilde{A}_{n}$ and the $\tilde{A}_{n}$ are separated from each other, each $J_{n}$ is isolated from the other $J_{m}$, i.e., for each $n$ there exists $\epsilon_{n}>0$ such that the $\epsilon_{n}$-neighborhood $B\left(J_{n}, \epsilon_{n}\right)$ does not meet any other $J_{m} \in \mathcal{J}$.

Let $C=C(0,2)$. Since $C \subset J(G)$ we see that $J(G)=\overline{G^{-1}(C)}$. We now show that for each $g \in G$, the set $g^{-1}(C) \subset J_{n}$ for some $n$. Write $g=g_{i_{1}} \circ \cdots \circ g_{i_{j}}$, where each $g_{i_{\ell}}$ is a generator for $G$. Suppose that $g_{i_{j}}=h_{n}$ for some $n$. Then, by the backward invariance of $A$ under each map in $G$, we have that $g^{-1}(C) \subset g^{-1}(A) \subset$ $h_{n}^{-1}(A) \subset A_{n}$. Since $g^{-1}(C)$ is connected, $g^{-1}(C)>B(0,1 / 2)$, and $J\left(k_{n}\right) \cup J\left(h_{n}\right)$ meets both the inner boundary and outer boundary of $A_{n}$, we must have that $g^{-1}(C)$ meets $J\left(k_{n}\right) \cup J\left(h_{n}\right)$ and thus $g^{-1}(C) \subset J_{n}$. Note that the same argument (using $A_{n}^{\prime}$ ) holds if $g_{i_{j}}=k_{n}$. Thus we have shown that $G^{-1}(C) \subset \bigcup_{n \in \mathbb{N}} J_{n}$. Since 
the $J_{n}$ are isolated from each other and accumulate only to $C$, it follows that $J(G)=\overline{G^{-1}(C)} \subset \overline{\bigcup_{n \in \mathbb{N}} J_{n}} \subset C \cup \bigcup_{n \in \mathbb{N}} J_{n}$, and so $\# \mathcal{J}=\aleph_{0}$. Then also note that we must have $J_{\min }=J_{1}$, and so $J_{\min }$ does have property $(\star \lambda)$.

We now show that $J^{\prime}<J^{\prime \prime}$ for $J^{\prime}, J^{\prime \prime} \in \mathcal{J}$ does not necessarily imply $g^{*}\left(J^{\prime}\right)<$ $g^{*}\left(J^{\prime \prime}\right)$. Indeed, we see that $h_{n}^{*}(J)=J_{n}$ for all $J \in \mathcal{J}$.

We note that we could easily adapt this example (by letting $b_{n}=0.5+1 / n$ ) to produce $G_{1} \in \mathcal{G}$ such that $J_{\min }\left(G_{1}\right)$ does not have property $(\star \lambda)$, but $J_{\max }$ does. Or we could produce $G_{2}=\left\langle G, G_{1}\right\rangle \in \mathcal{G}$ such that neither $J_{\min }$ nor $J_{\max }$ has property $(\star \lambda)$.

Note that in the above example(s) where $J_{\min }$ (respectively $J_{\max }$ ) did not meet $\bigcup_{\lambda \in \Lambda} J\left(g_{\lambda}\right)$, it was true that $J_{\min }$ (respectively $J_{\max }$ ) was contained in $\overline{\bigcup_{\lambda \in \Lambda} J\left(g_{\lambda}\right)}$. We will prove in Theorem 4.9 that this is indeed always the case. First we need to prove the following lemma.

Lemma 4.8. Let $\left\{g_{\lambda}\right\}_{\lambda \in \Lambda} \subset G \in \mathcal{G}_{\text {dis }}$. Let $\mathcal{C}$ denote the connected components of $\overline{\bigcup_{\lambda \in \Lambda} J\left(g_{\lambda}\right)}$. Then both $M^{\prime}=\min _{C \in \mathcal{C}} C$ and $M^{\prime \prime}=\max _{C \in \mathcal{C}} C$ exist (with respect to the surrounding order $\leq)$. Also, $P H(C) \supset \hat{K}(G) \supset P^{*}(G)$ for each $C \in \mathcal{C}$.

Proof. First we note that $\infty \in F(G)$ by Theorem 1.20, and so all sets in $\mathcal{C}$ are contained in $\mathbb{C}$. Let $C \in \mathcal{C}$. Suppose that $z_{0} \in \hat{K}(G) \backslash P H(C)$. Let $\gamma$ be a curve in $\overline{\mathbb{C}} \backslash P H(C)$ connecting $z_{0}$ to $\infty$ and set $\epsilon=\operatorname{dist}(\gamma, P H(C))$. By Corollary 2.18 , there exists $\lambda \in \Lambda$ such that $J\left(g_{\lambda}\right) \subset B(C, \epsilon)$. Hence, we see that $\gamma$ is outside $J\left(g_{\lambda}\right)$ implying that $g_{\lambda}^{n}\left(z_{0}\right) \rightarrow \infty$ and thus contradicting the fact that $z_{0} \in \hat{K}(G)$.

Lemma 2.3 shows that the compact connected sets in $\mathcal{C}$ are linearly ordered with respect to the surrounding order. The existence of $M^{\prime}$ and $M^{\prime \prime}$ then follows directly from Lemma 2.13

Theorem 4.9. Consider $G=\left\langle g_{\lambda}: \lambda \in \Lambda\right\rangle \in \mathcal{G}_{\text {dis }}$. Let $A=\bigcup_{\lambda \in \Lambda} J\left(g_{\lambda}\right)$ and denote by $M^{\prime}$ and $M^{\prime \prime}$ the minimal and maximal connected components of $\bar{A}$, respectively. Then both $J_{\min } \supset M^{\prime}$ and $J_{\max } \supset M^{\prime \prime}$ and, in particular, both $J_{\min } \cap \bar{A} \neq \emptyset$ and $J_{\max } \cap \bar{A} \neq \emptyset$. Furthermore, we have the following:

(1) If $J_{\min } \cap A=\emptyset$ (i.e., $J_{\min }$ does not have property $(\star \lambda)$ ), then $J_{\min }=M^{\prime}$ and $J_{\min }$ is the boundary of the unbounded component of $\mathbb{C} \backslash J_{\min }$.

(2) If $J_{\max } \cap A=\emptyset$ (i.e., $J_{\max }$ does not have property $(\star \lambda)$ ), then $J_{\max }=M^{\prime \prime}$ and $J_{\max }$ is the boundary of the bounded component of $\mathbb{C} \backslash J_{\max }$ which contains $J_{\min }$.

Remark 4.10. In the above theorem, if $J(G)$ is connected, then $J_{\min }=J(G)=J_{\max }$ meets all $J(g)$ such that $g \in G$ and thus meets $A$.

Open Question. We notice in Example 4.7 that $J_{\max } \cap A=\emptyset$ and $J_{\max }$ is a simple closed curve. However, it is not clear, in general, whether the hypothesis $J_{\max } \cap A=\emptyset$ for $G=\left\langle g_{\lambda}: \lambda \in \Lambda\right\rangle \in \mathcal{G}_{\text {dis }}$ must necessarily lead to the conclusion that $J_{\max }$ is a simple closed curve. It is also not clear under this hypothesis whether $J_{\max }$ must be the common boundary of exactly two complementary domains. So we state these as open questions (noting that the corresponding questions regarding $J_{\min }$ are also open).

Remark 4.11. We note that we could also use Theorem 4.9 to see that $J_{\min }$ in Example 4.7 has property $(\star \lambda)$, since $J_{\text {min }}$ must meet $\overline{\bigcup_{j=1}^{\infty} J\left(k_{j}\right) \cup J\left(h_{j}\right)}=C(0,2) \cup$ $\bigcup_{j=1}^{\infty}\left(J\left(k_{j}\right) \cup J\left(h_{j}\right)\right)$ but does not meet $C(0,2)$. 
Proof. Let $J^{\prime}, J^{\prime \prime} \in \mathcal{J}$ be such that $M^{\prime} \subset J^{\prime}$ and $M^{\prime \prime} \subset J^{\prime \prime}$. Fix $\lambda \in \Lambda$. By the minimality of $M^{\prime}$, we have either $J\left(g_{\lambda}\right) \subset J^{\prime}$ or $J\left(g_{\lambda}\right)>J^{\prime}$. Then $g_{\lambda}^{*}\left(J^{\prime}\right) \geq J^{\prime}$ by Lemmas 2.14 and 2.16 and Theorem 1.20. Since $g_{\lambda}^{*}\left(J^{\prime}\right) \geq J^{\prime}$ for all $\lambda \in \Lambda$ we must have that $J^{\prime}=J_{\min }$ (because the closed set $\bigcup_{\left\{J \in \mathcal{J}: J \geq J^{\prime}\right\}} J$ is then backward invariant under each $g \in G$ ). Similarly, we see that $J^{\prime \prime}=\bar{J}_{\max }$. Thus both $J_{\min } \cap$ $\bar{A} \neq \emptyset$ and $J_{\max } \cap \bar{A} \neq \emptyset$.

We now prove (2) by first showing that $J(G) \subset J_{\min } \cup M^{\prime \prime} \cup A n n\left(J_{\min }, M^{\prime \prime}\right)$. Fix $\lambda \in \Lambda$. Since $P H\left(M^{\prime \prime}\right) \supset P^{*}(G)$ by Lemma 4.8, we see that $g_{\lambda}^{-1}\left(M^{\prime \prime}\right)$ is connected using Lemma 2.8. Thus $g_{\lambda}^{-1}\left(M^{\prime \prime}\right) \cap M^{\prime \prime}=\emptyset$. Otherwise, $J\left(g_{\lambda}\right)$ meets $J_{\max }$ by Lemma 2.16 which violates our hypothesis that $J_{\max } \cap A=\emptyset$. Hence $g_{\lambda}^{-1}\left(M^{\prime \prime}\right)<M^{\prime \prime}$ by Lemma 2.14(b).

From the fact that $g_{\lambda}^{-1}\left(J_{\min }\right) \geq J_{\min }$ and $g_{\lambda}^{-1}\left(M^{\prime \prime}\right)<M^{\prime \prime}$ for all $\lambda \in \Lambda$, we deduce from Lemma 2.8 that the closed annulus-type region $A_{1}=J_{\min } \cup M^{\prime \prime} \cup$ $\operatorname{Ann}\left(J_{\min }, M^{\prime \prime}\right)$ is backward invariant under each generator (and thus under each $g \in G)$. Hence $J(G) \subset J_{\min } \cup M^{\prime \prime} \cup \operatorname{Ann}\left(J_{\min }, M^{\prime \prime}\right)$, as desired.

We now suppose there exists $w \in J_{\max } \backslash M^{\prime \prime}$. Such a point $w$ must then necessarily lie in $\operatorname{Ann}\left(J_{\min }, M^{\prime \prime}\right)$ ( since $w \in J_{\min }$ would imply $J_{\min }=J_{\max }=J(G)$, and thus $J_{\max }$ clearly meets $A$ ). Let $U$ be the connected component of $\mathbb{C} \backslash M^{\prime \prime}$ which contains $J_{\min }$. Note that $w \in U$ by definition of $A n n\left(J_{\min }, M^{\prime \prime}\right)$. Recall that $\partial \hat{K}(G) \subset J_{\min }$. Let $\gamma$ be a curve in $U$ which connects $w$ to some $z_{0} \in \hat{K}(G)$ and set $\epsilon=\operatorname{dist}\left(\gamma, M^{\prime \prime}\right)>0$. By Corollary 2.18 there exists a generator $g_{\lambda_{0}} \in G$ such that $J\left(g_{\lambda_{0}}\right) \subset B\left(M^{\prime \prime}, \epsilon\right)$. Thus $\gamma \cap J\left(g_{\lambda_{0}}\right)=\emptyset$. Since $\hat{K}(G) \subset P H\left(J\left(g_{\lambda_{0}}\right)\right)$, we see that $z_{0} \in \gamma \cap P H\left(J\left(g_{\lambda_{0}}\right)\right)$, and so $\gamma<J\left(g_{\lambda_{0}}\right)$. Hence $\{w\}<J\left(g_{\lambda_{0}}\right)$, which implies (by Corollary 2.10) either $J_{\max }<J\left(g_{\lambda_{0}}\right)$ or $J_{\max } \cap J\left(g_{\lambda_{0}}\right) \neq \emptyset$. Since neither of these can occur, we conclude that no such $w$ exists and thus $J_{\max }=M^{\prime \prime}$.

Recall that $U$ is the bounded component of $\mathbb{C} \backslash J_{\max }$ which contains $J_{\min }$. Since $J_{\max } \cap A=\emptyset$, we have that for every $\lambda \in \Lambda$, the set $J\left(g_{\lambda}\right)$ is contained in $U$. Hence $\bar{A} \subset \bar{U}$, and so $J_{\max }=M^{\prime \prime} \subset \bar{A} \subset \bar{U}$, which implies $J_{\max }=\partial U$.

The proof for case (1) is similar but simpler. In this case the point $\infty$ can play the role of $z_{0}$ in order to help demonstrate that any point in $J_{\min } \backslash M^{\prime}$ must lie "outside" of some $J\left(g_{\lambda}\right)$ (which is a contradiction). We omit the details.

Corollary 4.12. When $G=\left\langle g_{\lambda}: \lambda \in \Lambda\right\rangle \in \mathcal{G}$ with $\overline{\bigcup_{\lambda \in \Lambda} J\left(g_{\lambda}\right)}=\bigcup_{\lambda \in \Lambda} J\left(g_{\lambda}\right)$, then both $J_{\min }$ and $J_{\max }$ must have property $(\star \lambda)$.

Remark 4.13. The above corollary applies, for example, when $G=\left\langle g_{\lambda}: \lambda \in \Lambda\right\rangle \in$ $\mathcal{G}_{\text {dis }}$ has $\bigcup_{\lambda \in \Lambda} J\left(g_{\lambda}\right)=\bigcup_{k=1, \ldots, n} J\left(g_{\lambda_{k}}\right)$. Such non-compactly generated examples can easily be constructed. Other more "exotic" examples can also be constructed to satisfy the hypotheses of the corollary.

Example 4.14. We note that without the hypothesis that $J_{\min } \cap A=\emptyset$ in Theorem 4.9 (1), the conclusion that $J_{\min }=M^{\prime}$ might not hold. Set $f_{1}(z)=z^{2}, f_{2}(z)=$ $(z-\epsilon)^{2}+\epsilon$, and $f_{3}(z)=z^{2} / 4$. For $\epsilon>0$ small and $m_{1}, m_{2}, m_{3}$ all large we set $g_{1}=f_{1}^{m_{1}}, g_{2}=f_{2}^{m_{2}}$, and $g_{3}=f_{3}^{m_{3}}$ and note that $G=\left\langle g_{1}, g_{2}, g_{3}\right\rangle \in \mathcal{G}_{d i s}$. Thus $M^{\prime}=J\left(f_{1}\right) \cup J\left(f_{2}\right)=C(0,1) \cup C(\epsilon, 1)$. However, the real point in $g_{1}^{-1}(\{1+\epsilon\})$ is clearly in $g_{1}^{-1}\left(J\left(f_{2}\right)\right) \subset J_{\min }$ but not in $M^{\prime}$.

Theorem 4.15. Let $G=\left\langle g_{\lambda}: \lambda \in \Lambda\right\rangle \in \mathcal{G}_{\text {dis }}$ and suppose $J_{\min } \cap \bigcup_{\lambda \in \Lambda} J\left(g_{\lambda}\right)=\emptyset$. Then $\partial \hat{K}(G) \subset M^{\prime} \subset \overline{\bigcup_{\lambda \in \Lambda} J\left(g_{\lambda}\right)}$, where $M^{\prime}$ is the minimal connected component of $\overline{\bigcup_{\lambda \in \Lambda} J\left(g_{\lambda}\right)}$. 
Proof. By Theorem 4.9(1), we see that $\partial \hat{K}(G) \subset J(G) \subset M^{\prime} \cup J_{\max } \cup A n n\left(M^{\prime}, J_{\max }\right)$. Moreover, by Lemma 4.8, we have $\partial \hat{K}(G) \subset P H\left(M^{\prime}\right)$. Hence $\partial \hat{K}(G) \subset M^{\prime}$.

Having discussed properties $(\star)$ and $(\star \lambda)$ with respect to $J_{\min }$ and $J_{\max }$, we now turn our attention to a general $J \in \mathcal{J}$. In particular, we investigate what can be said about which $J \in \mathcal{J}$ have property $(\star)$ or $(\star \lambda)$. We also concern ourselves with the question of when does every $J \in \mathcal{J}$ have property $(\star)$ or $(\star \lambda)$. We begin with the following definition.

Definition 4.16. Let $G \in \mathcal{G}$. We say that $J \in \mathcal{J}$ is isolated in $\mathcal{J}$ if there exists $\epsilon>0$ such that $B(J, \epsilon)$ does not meet any other set in $\mathcal{J}$.

Lemma 4.17. Let $G \in \mathcal{G}$ with $J \in \mathcal{J}$ isolated in $\mathcal{J}$. Then $J$ has property $(\star)$.

Proof. Assume that $\epsilon>0$ is such that $B(J, \epsilon)$ does not meet any other set in $\mathcal{J}$. Since $J(G)=\overline{\bigcup_{g \in G} J(g)}$ by Theorem 1.2, we see that any point in $J$ must have, within a distance $\epsilon$, a point in some $J(g)$, where $g \in G$. It must then be the case that $J(g)$, which lies in some set in $\mathcal{J}$, must lie entirely in $J$.

Remark 4.18. If $G \in \mathcal{G}$ is such that $\# \mathcal{J}<+\infty$, then clearly each $J \in \mathcal{J}$ is isolated in $\mathcal{J}$, and so each $J \in \mathcal{J}$ has property $(\star)$. We note, however, that if each $J \in \mathcal{J}$ is isolated in $\mathcal{J}$, then it is not necessarily the case that each $J \in \mathcal{J}$ has property $(\star \lambda)$. See the proof of Theorem 1.23 where, for any positive integer $k$, a semigroup $G^{\prime} \in \mathcal{G}$ is constructed such that $\# \mathcal{J}=k$, but only $J_{\min }$ and $J_{\max }$ have property $(\star \lambda)$.

Remark 4.19. If $G=\left\langle h_{\lambda}: \lambda \in \Lambda\right\rangle \in \mathcal{G}$ where $\# \Lambda \leq \aleph_{0}$ and $\# \mathcal{J}$ is uncountable, then since $\# G=\aleph_{0}$ we see that some $J \in \mathcal{J}$ must fail to have property $(\star)$. An example of this is the Cantor set of circles in Example 1.19.

Example 4.20. Suppose $G \in \mathcal{G}$ and $\# \mathcal{J}=\aleph_{0}$. Then it is possible that not all $J \in \mathcal{J}$ have property $(\star)$ as in Example 4.7 However, it is also possible that all $J \in \mathcal{J}$ do have property $(\star)$ as in [29], Theorem 2.26 , where both $J_{\min }$ and $J_{\max }$ have property $(\star \lambda)$ and each other component of $J(G)$ is isolated in $\mathcal{J}$.

We saw above that isolated $J \in \mathcal{J}$ have property $(\star)$. We now show that this is also true for the components of $J(G)$ which contain the pre-image of an isolated $J \in \mathcal{J}$.

Claim 4.21. Let $G \in \mathcal{G}$. If $J_{1} \in \mathcal{J}$ is isolated in $\mathcal{J}$ and $h^{-1}\left(J_{1}\right) \subset J \in \mathcal{J}$ for some $h \in G$, then $J$ has property $(\star)$.

Proof. Since $J_{1}$ is isolated in $\mathcal{J}$, Lemma 4.17 implies there exists $g \in G$ such that $J(g) \subset J_{1}$. Thus, since $J_{1}$ is isolated in $\mathcal{J}$, for large $n \in \mathbb{N}$ we have $g^{-n}(J) \subset J_{1}$. Hence $h^{-1}\left(g^{-n}(J)\right) \subset h^{-1}\left(J_{1}\right) \subset J$, which implies $J\left(g^{n} \circ h\right) \subset J$.

Open Question. If $\# \mathcal{J}=\aleph_{0}$ and $G \in \mathcal{G}$ is finitely generated, then must every $J \in \mathcal{J}$ have property $(\star)$ ? Note that the finitely generated condition is required by Example 4.7 Also, Example 1.19 shows that if $\# \mathcal{J}$ is uncountable, $\mathcal{J}$ can have (uncountably many) $J$ which fail to have property $(\star$ ).

We now turn our attention to considering those semigroups where $J_{\min }$ has property $(\star \lambda)$. In particular, we examine the generating maps whose Julia sets meet $J_{\min }$ as well as the subsemigroup generated by just these special maps. 
Definition 4.22. Let $G=\left\langle h_{\lambda}: \lambda \in \Lambda\right\rangle \in \mathcal{G}_{\text {dis }}$. We set

$$
B_{\min }=B_{\min }(G):=\left\{\lambda \in \Lambda: J\left(h_{\lambda}\right) \subset J_{\min }(G)\right\} .
$$

If $B_{\min } \neq \emptyset$, let $H_{\min }(G)$ be the subsemigroup of $G$ which is generated by $\left\{h_{\lambda}: \lambda \in\right.$ $\left.B_{\min }\right\}$.

The following proposition has been shown in [29, 33 .

Proposition 4.23. Let $G=\left\langle h_{\lambda}: \lambda \in \Lambda\right\rangle \in \mathcal{G}_{\text {dis }}$. If $\left\{h_{\lambda}: \lambda \in \Lambda\right\}$ is compact in Poly, then $B_{\min }$ is a proper non-empty subset of $\Lambda$ under the above notation.

Proof. The result follows since by Proposition 4.1 both $J_{\min }$ and $J_{\max }$ have property $(\star \lambda)$.

It is natural to investigate the relationship between $H_{\min }(G)$ and $G$. Specifically we ask, and answer, the following questions for a semigroup $G \in \mathcal{G}_{d i s}$ :

(1) Must $J\left(H_{\min }(G)\right)=J_{\min }(G)$ ?

(2) Must $J\left(H_{\min }(G)\right)$ be connected?

(3) Must $J_{\min }\left(H_{\min }(G)\right)=J_{\min }(G)$ ?

(4) Must $H_{\min }\left(H_{\min }(G)\right)=H_{\min }(G)$ ?

The answer to each of these questions is $\mathrm{NO}$, as we see in this next example.

Example 4.24. We will construct a single 3-generator polynomial semigroup $G \in$ $\mathcal{G}_{\text {dis }}$ which negatively answers questions (1)-(4). Furthermore, we will show that $\# \mathcal{J}=\aleph_{0}$.

Let $h_{1}(z)=-z^{2}$ and $f_{2}(z)=z^{2} / \sqrt{2}$ and note that $J\left(h_{1}\right)=C(0,1)$ and $J\left(f_{2}\right)=$ $C(0, \sqrt{2})$. We set $h_{2}=f_{2}^{m_{2}}$, where conditions on the large $m_{2} \in \mathbb{N}$ will be specified later. Choose point $P \in h_{2}^{-1}\left(J\left(h_{1}\right)\right)$ such that $P>0$. Note that $P=\sqrt{2}-\delta$, where $\delta$ is small for $m_{2}$ large. Hence $P^{2}=2-2 \sqrt{2} \delta+\delta^{2}$ and $P^{4}=4-8 \sqrt{2} \delta+O\left(\delta^{2}\right)$.

Setting $r=\frac{P^{4}+P^{2}}{2}=3-M_{1} \delta+O\left(\delta^{2}\right)$ and $\epsilon=\frac{P^{4}-P^{2}}{2}=1-M_{2} \delta+O\left(\delta^{2}\right)$ where $M_{1}, M_{2}>0$, we see that for $f_{3}(z)=\frac{(z-\epsilon)^{2}}{r}+\epsilon$ both $h_{1}(P)=-P^{2}=\epsilon-r$ and $h_{1}^{2}\left(e^{i \pi / 4} P\right)=P^{4}=r+\epsilon$ lie in $C(\epsilon, r)=J\left(f_{3}\right)$.

Suppose there exists $w \in h_{1}^{-1}\left(J\left(f_{3}\right)\right) \cap J\left(f_{3}\right)$, i.e., $h_{1}(w) \in J\left(f_{3}\right)$ and $w \in J\left(f_{3}\right)$. Then $\left|h_{1}(w)\right|=|w|^{2} \geq\left|-P^{2}\right|^{2}=P^{4}$ since $-P^{2}$ is the point in $J\left(f_{3}\right)$ of smallest modulus. Since $P^{4}$ is the point in $J\left(f_{3}\right)$ of largest modulus, we see that $h_{1}(w)$ could only be in $J\left(f_{3}\right)$ if $w=-P^{2}$. However, $h_{1}\left(-P^{2}\right)=-P^{4} \notin J\left(f_{3}\right)$, and so we have $h_{1}^{-1}\left(J\left(f_{3}\right)\right) \cap J\left(f_{3}\right)=\emptyset$ and thus $h_{1}^{-1}\left(J\left(f_{3}\right)\right)<J\left(f_{3}\right)$ (see Figure 1 ).

Since for $m_{2}$ large we clearly have $h_{2}^{-1}\left(J\left(f_{3}\right)\right)<J\left(f_{3}\right)$, we are then free to choose $A^{\prime}$ to be any closed annulus such that $A^{\prime} \subset B(\epsilon, r)$, int $\left(A^{\prime}\right) \neq \emptyset$, and $A^{\prime}>$ $h_{1}^{-1}\left(J\left(f_{3}\right)\right) \cup h_{2}^{-1}\left(J\left(f_{3}\right)\right)$.

Choose $m_{3} \in \mathbb{N}$ large enough so that $h_{3}=f_{3}^{m_{3}}$ maps $A^{\prime}$ into $B(0,1)$ (which can be done since the super attracting fixed point of $f_{3}$ is $\epsilon \in B(0,1)$ ). This then implies that $B(0,1)$ is forward invariant under each map $h_{1}, h_{2}$ and $h_{3}$ and that $P^{*}(G) \subset B(0,1)=\operatorname{int} \hat{K}(G) \subset F(G)$, where $G=\left\langle h_{1}, h_{2}, h_{3}\right\rangle$.

Let $A=J\left(h_{2}\right) \cup h_{2}^{-1}\left(J\left(h_{1}\right)\right) \cup h_{1}^{-1}\left(J\left(h_{3}\right)\right) \subset J(G)$. Because $h_{1}^{-1}\left(J\left(h_{3}\right)\right)$ is connected (Corollary 2.9) and contains both the point $i P^{2}$, which lies outside the circle $J\left(h_{2}\right)$, and the point $P \in h_{2}^{-1}\left(J\left(h_{1}\right)\right)$, which lies inside the circle $J\left(h_{2}\right)$, we see that the set $A$ is connected.

Note that $e^{i \pi / 4} P \in h_{2}^{-1}\left(J\left(h_{1}\right)\right) \subset A$ and $e^{i \pi / 4} P \in h_{1}^{-1}\left(h_{1}^{-1}\left(J\left(h_{3}\right)\right)\right) \subset h_{1}^{-1}(A)$ since $h_{1}^{2}\left(e^{i \pi / 4} P\right)=P^{4} \in J\left(h_{3}\right)$. Thus $h_{1}^{-1}(A) \cap A \neq \emptyset$, and since $h_{1}^{-n}(A)$ is 


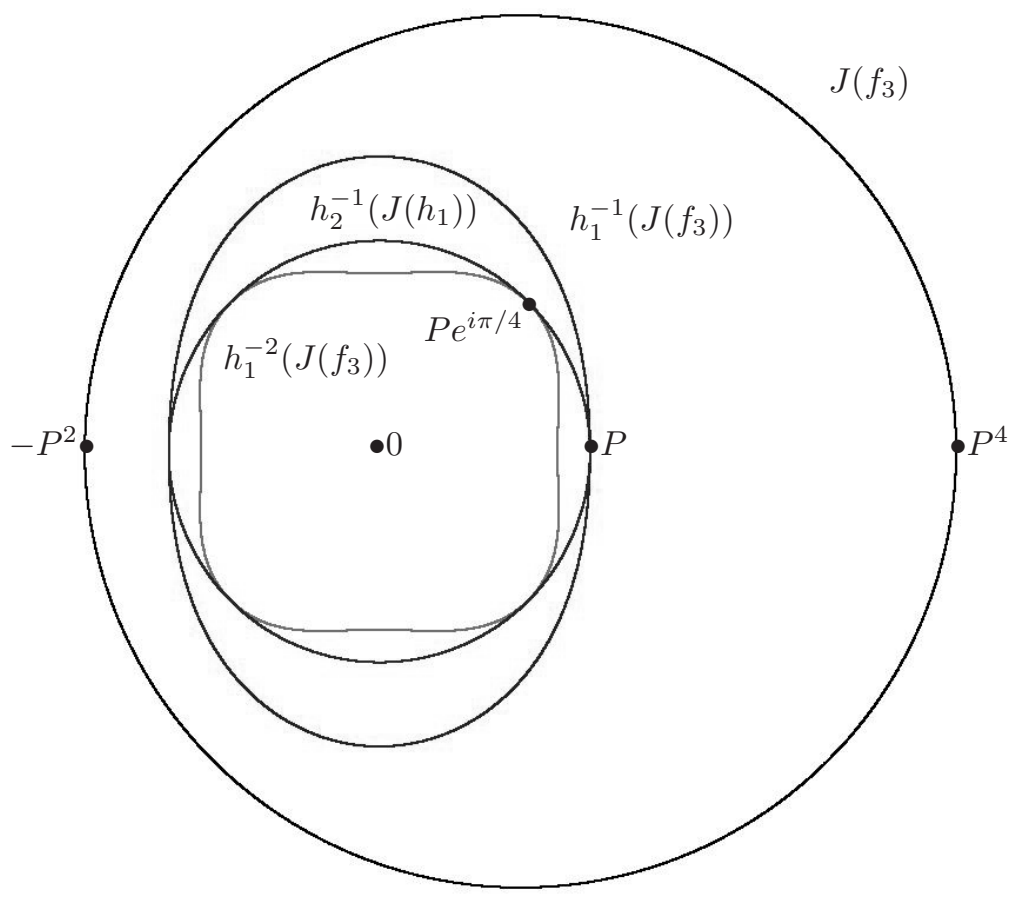

Figure 2. This figure shows the sets (from outside to inside) $J\left(f_{3}\right), h_{1}^{-1}\left(J\left(f_{3}\right)\right), h_{2}^{-1}\left(J\left(h_{1}\right)\right)$, and $h_{1}^{-2}\left(J\left(f_{3}\right)\right)$. For this picture we chose $m_{2}=5$ and $P=2^{31 / 64} \approx 1.39898$.

connected for each $n \in \mathbb{N}$ by Lemma 2.8, we see that Lemma 2.16 implies that $A$ and $J\left(h_{1}\right)$ are contained in a single $J \in \mathcal{J}$. Since $P^{*}(G) \subset B(0,1) \subset F(G)$, we see that $J=J_{\min }$. Thus both $J\left(h_{1}\right)$ and $J\left(h_{2}\right)$ are contained in $J_{\min }(G)$. Note that $\partial \hat{K}(G)=J\left(h_{1}\right) \neq J_{\min }(G)$.

Both $h_{1}$ and $h_{2}$ map $A^{\prime}$ into the unbounded component of $\overline{\mathbb{C}} \backslash J\left(h_{3}\right)$ (since $A^{\prime}$ is outside of both $h_{1}^{-1}\left(J\left(h_{3}\right)\right)$ and $\left.h_{2}^{-1}\left(J\left(h_{3}\right)\right)\right)$, which is forward invariant under each map $h_{1}, h_{2}$ and $h_{3}$. The map $h_{3}$ maps $A^{\prime}$ into $B(0,1)$, which is also forward invariant under each map $h_{1}, h_{2}$ and $h_{3}$. Hence for any $g \in G$ we have that $g\left(A^{\prime}\right) \cap A^{\prime}=\emptyset$, and so $\operatorname{int}\left(A^{\prime}\right) \subset F(G)$. We conclude that $J\left(h_{3}\right)$ is not contained in $J_{\min }$.

Thus we have that $H_{\min }(G)=\left\langle h_{1}, h_{2}\right\rangle$. One can easily show that $J\left(H_{\min }(G)\right)$ is disconnected (Cantor set of circles), and thus $J\left(H_{\min }(G)\right) \neq J_{\min }(G)$. Also $H_{\min }\left(H_{\min }(G)\right)=\left\langle h_{1}\right\rangle \neq\left\langle h_{1}, h_{2}\right\rangle=H_{\min }(G)$ and $J_{\min }(G) \neq J_{\min }\left(H_{\min }(G)\right)$.

We now show $\# \mathcal{J}_{G}=\aleph_{0}$. Consider the set $B=J_{\min }(G) \cup \bigcup_{n=1}^{\infty} h_{3}^{-n}\left(J_{\min }(G)\right) \cup$ $J\left(h_{3}\right) \subset J(G)$, which clearly contains more than three points. We have $J\left(h_{3}\right) \subset$ $J_{\max }(G)$ and $h_{1}^{-1}\left(J\left(h_{3}\right)\right) \subset J_{\min }(G)$. By Theorem 1.14 or Corollary 2.9, we obtain $h_{1}^{-1}(B) \subset h_{1}^{-1}(J(G)) \subset J_{\min }(G) \subset B$. Similarly, taking $m_{2}$ so large, we may assume $h_{2}^{-1}\left(J\left(h_{3}\right)\right) \subset J_{\min }(G)$, and it implies that $h_{2}^{-1}(B) \subset h_{2}^{-1}(J(G)) \subset$ $J_{\text {min }}(G) \subset B$. Since $B$ is closed and backward invariant under each generator of $G$ (and hence under every $g \in G$ ), we must have that $B=J(G)$. Also, since $h_{3}^{-1}\left(J_{\min }(G)\right)$ is connected (by Corollary 2.9) and does not meet $J_{\min }(G)$, we see 
that $J_{\min }(G)<h_{3}^{-1}\left(J_{\min }(G)\right)$. Repeated application of Lemma 2.8 shows us that $J_{\min }(G)<h_{3}^{-1}\left(J_{\min }(G)\right)<h_{3}^{-2}\left(J_{\min }(G)\right)<\ldots<h_{3}^{-n}\left(J_{\min }(G)\right)<\ldots$ From this we may conclude that $\mathcal{J}=\left\{J_{\min }(G), J\left(h_{3}\right), h_{3}^{-n}\left(J_{\min }(G)\right): n \in \mathbb{N}\right\}$, thus demonstrating that $\# \mathcal{J}_{G}=\aleph_{0}$.

Remark 4.25. Note that Example 4.24 does not settle (in the negative) the open question stated above since Claim 4.21 with $J_{1}=J_{\min }$ shows that each $J \in \mathcal{J}_{G}$ contains $J(g)$ for some $g \in G$. One could also note that every $J \in \mathcal{J}_{G}$ other than $J\left(h_{3}\right)$ is isolated in $\mathcal{J}_{G}$, and so from Lemma 4.17 each such $J$ has property $(\star)$.

Question. Does there exist an example of some $G \in \mathcal{G}$ which can negatively answer questions (1)-(4) addressed by Example 4.24 but where $\# \mathcal{J}_{G}$ is finite? The answer, as we see in the next example, is YES. We will also see that this example will settle two other questions that naturally arise when considering the two following results. In [29, 33] it was shown that, for each positive integer $k$, there exists a semigroup $G \in \mathcal{G}_{\text {dis }}$ with $2 k$ generators such that $J(G)$ has exactly $k$ components. Furthermore, in 25] it was shown that any semigroup in $\mathcal{G}$ generated by exactly three elements will have a Julia set with either one or infinitely many components. Hence we have the following questions.

(5) What is the fewest number of generators that can produce a semigroup $G \in \mathcal{G}_{\text {dis }}$ with $\# \mathcal{J}=3$ ?

(6) For fixed integer $k>3$, what is the fewest number of generators that can produce a semigroup $G \in \mathcal{G}_{\text {dis }}$ with $\# \mathcal{J}=k$ ?

The answer to both of these questions is four, as stated in Theorem 1.23 whose proof is now given.

Proof of Theorem 1.23. Fix $k \geq 2$ since the $k=1$ case is trivial. Let maps $h_{1}, f_{2}$, and $f_{3}$ and integer $m_{2} \in \mathbb{N}$ be defined as in Example 4.24. Again, we set $h_{2}=f_{2}^{m_{2}}$ and $h_{3}=f_{3}^{m_{3}}$, where large $m_{3} \in \mathbb{N}$ will be specified to fit the stipulations given below. Letting $\gamma_{1}$ denote $C(0,1)$ and $\gamma_{2}$ denote the boundary of the unbounded component of $\mathbb{C} \backslash\left(h_{1}^{-1}\left(J\left(f_{3}\right)\right) \cup h_{2}^{-1}\left(J\left(f_{3}\right)\right)\right)$, we set $B=\overline{A n n\left(\gamma_{1}, \gamma_{2}\right)}$. Let $A^{\prime}$ be any closed annulus such that $A^{\prime} \subset B(\epsilon, r), \operatorname{int}\left(A^{\prime}\right) \neq \emptyset$, and $A^{\prime}>B$. We choose $m_{3} \in \mathbb{N}$ large enough so that $h_{3}^{-1}(B)>A^{\prime}>B$. Set $G=\left\langle h_{1}, h_{2}, h_{3}\right\rangle$ and note that for $m_{3}$ large enough, $\gamma_{1} \cup \gamma_{2} \subset J_{\min }(G) \subset B$, as in Example 4.24.

Set $\ell=k-2$. Let $C=C\left(\epsilon_{0}, r_{0}\right)$ be the circle which is internally tangent to the circle $J\left(h_{3}\right)$ at the point $\epsilon+r=P^{4}$ such that $C$ meets $h_{3}^{-(\ell+1)}(B)$ and $\overline{B\left(\epsilon_{0}, r_{0}\right)} \supset$ $h_{3}^{-(\ell+1)}(B)$. Hence $C$ must necessarily meet $h_{3}^{-(\ell+1)}\left(\gamma_{2}\right) \subset h_{3}^{-(\ell+1)}\left(J_{\min }(G)\right)$ and $C>h_{3}^{-\ell}(B)>\cdots>h_{3}^{-1}(B)>B$. Note that as $m_{3} \rightarrow \infty$, we have $\epsilon_{0} \rightarrow \epsilon$ and $r_{0} \rightarrow r$. We may then assume that $m_{3}$ has been chosen large enough so that $\epsilon_{0} \in B(0,1)$.

Set $f_{4}(z)=\frac{\left(z-\epsilon_{0}\right)^{2}}{r_{0}}+\epsilon_{0}$ and observe that $J\left(f_{4}\right)=C$. Let $R>0$ be large enough so that $f_{j}(\mathbb{C} \backslash \overline{B(0, R)}) \subset \mathbb{C} \backslash \overline{B(0, R)}$ for $j=1, \ldots, 4$ (where $f_{1}:=h_{1}$ ). Let $A^{\prime \prime}$ be a closed annulus such that $\operatorname{int}\left(A^{\prime \prime}\right) \neq \emptyset$ and $h_{3}^{-\ell}(B)<A^{\prime \prime}<h_{3}^{-(\ell+1)}(B)$. Then $h_{3}^{\ell+1}\left(A^{\prime \prime}\right) \subset B(0,1)$. Let $A_{0}:=A^{\prime} \cup \bigcup_{j=0}^{\ell} h_{3}^{j}\left(A^{\prime \prime}\right)$. We define $h_{4}=f_{4}^{m_{4}}$, where $m_{4} \in \mathbb{N}$ is large enough such that (i) $h_{4}\left(A_{0}\right) \subset B(0,1)$, (ii) $h_{4}^{-1}\left(\gamma_{1}\right)$ meets $h_{3}^{-(\ell+1)}\left(J_{\min }(G)\right)$ (this is possible since the connected set $h_{3}^{-(\ell+1)}\left(J_{\min }(G)\right)$ meets, but is not contained in, $C)$, (iii) $h_{4}\left(\bigcup_{j=1}^{2} \bigcup_{h \in\left\langle h_{1}, h_{2}, h_{3}\right\rangle \cup\{i d\}} h \circ h_{j}\left(A_{0}\right)\right) \subset \mathbb{C} \backslash \overline{B(0, R)}$ 
(note that $\overline{h_{1}\left(A_{0}\right) \cup h_{2}\left(A_{0}\right)} \subset \overline{\mathbb{C}} \backslash K\left(h_{3}\right)$, which is equal to the connected component of $F\left(\left\langle h_{1}, h_{2}, h_{3}\right\rangle\right)$ containing $\left.\infty\right)$, and (iv) $h_{4}^{-1}\left(\gamma_{1}\right)>h_{3}^{-\ell}(B)$.

Set $G^{\prime}=\left\langle h_{1}, h_{2}, h_{3}, h_{4}\right\rangle$. Since $B(0,1)$ is forward invariant under each map in $G^{\prime}$, we conclude that $B(0,1) \subset F\left(G^{\prime}\right)$ and $P^{*}\left(G^{\prime}\right) \subset B(0,1)$. Thus $G^{\prime} \in \mathcal{G}$. Also, $\operatorname{int}\left(A_{0}\right) \subset F\left(G^{\prime}\right)$ since one can show that $g\left(A_{0}\right) \subset A_{0} \cup B(0,1) \cup \hat{\mathbb{C}} \backslash K\left(h_{3}\right)$ for all $g \in G^{\prime}$. Hence $G^{\prime} \in \mathcal{G}_{d i s}$. By applying Lemma 2.16 and Lemma 2.14 (noting that $\left.J_{\min }\left(G^{\prime}\right)<\operatorname{int}\left(A^{\prime}\right)<J\left(h_{3}\right)\right)$, we have that $h_{3}^{-n}\left(J_{\min }\left(G^{\prime}\right)\right)<h_{3}^{-(n+1)}\left(J_{\min }\left(G^{\prime}\right)\right)$ for all $n \geq 0$. Further, $J_{\max }\left(G^{\prime}\right)$, which must contain $J\left(h_{3}\right)$ and $J\left(h_{4}\right)$ by Proposition 4.1. must also contain $h_{3}^{-n}\left(J_{\min }\left(G^{\prime}\right)\right)$ for all $n>\ell$. By examining the dynamics one can then show that $J\left(G^{\prime}\right)=J_{\min }\left(G^{\prime}\right) \cup J_{\max }\left(G^{\prime}\right) \cup h_{3}^{-1}\left(J_{\min }\left(G^{\prime}\right)\right) \cup \cdots \cup$ $h_{3}^{-\ell}\left(J_{\min }\left(G^{\prime}\right)\right)$, since this set is closed and backward invariant under each generator of $G^{\prime}$. Moreover, since $h_{3}^{-\ell}\left(\gamma_{1}\right)<A^{\prime \prime}<h_{3}^{-(\ell+1)}\left(\gamma_{1}\right), h_{3}^{-\ell}\left(\gamma_{1}\right) \subset h_{3}^{-\ell}\left(J_{\min }\left(G^{\prime}\right)\right)$, $h_{3}^{-(\ell+1)}\left(\gamma_{1}\right) \subset J_{\max }\left(G^{\prime}\right)$ and $\operatorname{int}\left(A^{\prime \prime}\right) \subset F\left(G^{\prime}\right)$, we have $h_{3}^{-\ell}\left(J_{\min }\left(G^{\prime}\right)\right)<\operatorname{int}\left(A^{\prime \prime}\right)<$ $J_{\max }\left(G^{\prime}\right)$. Thus we see that $J\left(G^{\prime}\right)$ has exactly $k$ components.

Remark 4.26. The addition of one generating function in the proof of Theorem 1.23 to the semigroup in Example 4.24illustrates something of a general principle (which we decline to attempt to make precise) at work when dealing with the dynamics of semigroups in $\mathcal{G}_{\text {dis }}$. Namely, if one adds a generator (or a whole family of generators) whose Julia set does not meet $J_{\min }$ of the new larger semigroup, then key properties of the dynamics can often be preserved. See for example Theorems 1.27 and 1.29.

However, as we see in this next lemma, adding "too many" new functions will necessarily destroy certain critical aspects of the dynamics. In particular, if we look to produce a new semigroup in $\mathcal{G}$ by adding "too many" generating polynomials of small degree (such that $G_{k}$ defined in the lemma is not pre-compact) to a semigroup $G \in \mathcal{G}_{d i s}$, then the new semigroup will necessarily have a connected Julia set.

Lemma 4.27. Let $G=\left\langle h_{\lambda}: \lambda \in \Lambda\right\rangle \in \mathcal{G}_{\text {dis }}$. Then each $G_{k}=\{g \in G: \operatorname{deg}(g) \leq k\}$ is pre-compact in Poly and, in particular, each $\left\{h_{\lambda}: \lambda \in \Lambda\right\} \cap G_{k}$ is pre-compact in Poly.

Remark 4.28. As stated earlier, a possibly generating set for $G$ is $G$ itself, which is necessarily not pre-compact (since it contains elements of arbitrarily high degree). Thus it is impossible to strengthen Lemma 4.27 to conclude that $\left\{h_{\lambda}: \lambda \in \Lambda\right\}$ is pre-compact.

Proof. Note that $J(G)$ is bounded in $\mathbb{C}$ since Theorem 1.20 yields $\infty \in F(G)$. Choose $R>0$ such that $J(G) \subset \overline{B(0, R)}$. Then $\operatorname{Cap}(J(g)) \leq R$ for all $g \in G$, where $\operatorname{Cap}(E)$ denotes the logarithmic capacity of the set $E$ (see 1 for the definition and properties). Also, since $G \in \mathcal{G}_{\text {dis }}$ we have $\operatorname{int}(\hat{K}(G)) \neq \emptyset$ (see Theorem 1.20 or 29]), and so there exists a ball of some radius $r>0$ in $\hat{K}(G)$. Thus $\operatorname{Cap}(J(g)) \geq r$ for all $g \in G$.

Let $H_{n}=\{g \in G: \operatorname{deg}(g)=n\}$. In order to show that $G_{k}=\bigcup_{n=1}^{k} H_{n}$ is pre-compact, it suffices to show that each $H_{n}$ is pre-compact. We now fix $g(z)=$ $a_{n} z^{n}+\cdots+a_{0}$ in $H_{n}$ and proceed to show that $\left|a_{n}\right|$ is uniformly bounded below by $R^{1-n}$ and uniformly bounded above by $r^{1-n}$, and that the remaining coefficients $a_{n-1}, \ldots, a_{0}$ of $g(z)$ are uniformly bounded (above) by positive constants which only depend on $r, R$ and $n$. Recalling Remark 1.25, it then follows that $H_{n}$ is pre-compact. Since $\left|a_{n}\right|^{-1 /(n-1)}=\operatorname{Cap}(J(g))$ (see [8], p. 35), we see that $r^{1-n} \geq$ $\left|a_{n}\right| \geq R^{1-n}$. Express $g^{\prime}(z)=\beta\left(z-\alpha_{1}\right) \cdots\left(z-\alpha_{n-1}\right)$, where $\beta=n a_{n}$ and the 
$\alpha_{j}$ are the critical points of $g$ which, since $G \in \mathcal{G}$ and $\infty \in F(G)$, must lie in $\mathbb{C} \backslash F_{\infty}(G) \subset \overline{B(0, R)}$, where $F_{\infty}(G)$ denotes the connected component of $F(G)$ containing $\infty$.

One can multiply out the terms in the expansion of $g^{\prime}(z)$ and find an antiderivative to see that the $a_{n-1}, \ldots, a_{1}$ coefficients of $g(z)$ are also bounded by constants which depend only on $r, R$ and $n$. Now fix $z_{0} \in \hat{K}(G)$. Since $g(\hat{K}(G)) \subset$ $\hat{K}(G) \subset \overline{B(0, R)}$, we have $\left|g\left(z_{0}\right)\right|=\left|a_{n} z_{0}^{n}+\cdots+a_{0}\right| \leq R$. Thus, since $\left|a_{n}\right|, \ldots,\left|a_{1}\right|$ are bounded by constants depending only on $r, R$ and $n$, the same is true for $\left|a_{0}\right|$.

Remark 4.29. The proof of Lemma 4.27 also holds for any $G \in \mathcal{G}$ such that there exist both lower and upper bounds on $\operatorname{Cap}(J(g))$ for all $g \in G$ (e.g., when $\hat{K}(G)$ contains some non-degenerate continuum and $\infty \in F(G)$ ).

\section{Proof of Theorems 1.27 and 1.29}

Example 5.1. Let $f_{1}(z)=z^{2}+c$, where $c>0$ is small (thus $J\left(f_{1}\right)$ is a quasicircle). Let $z_{0} \in \mathbb{R}$ denote the finite attracting fixed point of $f_{1}$. Note that $f_{1}^{k}(0)$ increases to $z_{0}$. Choose $f_{2}(z)=\frac{\left(z-z_{0}\right)^{2}}{\left(c-z_{0}\right)}+z_{0}$ and note that $J\left(f_{2}\right)=C\left(z_{0},\left|c-z_{0}\right|\right)$. For $m_{1}, m_{2} \in \mathbb{N}$ large $h_{1}=f_{1}^{m_{1}}$ and $h_{2}=f_{2}^{m_{2}}$ each map $B\left(z_{0},\left|c-z_{0}\right|\right)$ into itself and $J(G)$ is disconnected for $G=\left\langle h_{1}, h_{2}\right\rangle$. Note that $P^{*}(G) \subset \overline{B\left(z_{0},\left|c-z_{0}\right|\right)}$, and so $G \in \mathcal{G}$. We have that $H=\left\langle h_{2}\right\rangle$ is hyperbolic, but since $f_{1}(0)=c \in J\left(h_{2}\right) \subset J(G)$, the semigroup $G=\left\langle H, h_{1}\right\rangle$ is not hyperbolic even though $J\left(h_{1}\right) \cap J_{\min }(G)=\emptyset$.

By conjugating $h_{2}$ by a suitable rotation we may assume that $\left\{h_{2}^{k}(c): k \in \mathbb{N}\right\}$ is dense in $J\left(h_{2}\right)$, and therefore we see that $H$ can be hyperbolic and have $G$ fail to even be subhyperbolic. However, Theorem 1.27 does imply that $G=\left\langle H, h_{1}\right\rangle$ is semihyperbolic.

Remark 5.2. In contrast to the analogous behavior of Iterated Function Systems where contraction in each generating map leads to a semigroup (IFS) that is overall contracting, we see that in Example 5.1 each map of the semigroup $G$ is hyperbolic, yet the entire semigroup $G$ fails to be hyperbolic. To see this, note that each map $h_{2}^{n}$ is hyperbolic and for each map $g \in G \backslash\left\{h_{2}^{n}\right\}$ we have $P^{*}(g) \subset P^{*}(G) \subset$ $\overline{B\left(z_{0},\left|c-z_{0}\right|\right)}$ and $J(g)>\overline{B\left(z_{0},\left|c-z_{0}\right|\right)}$, which implies $g$ is hyperbolic.

We now state a lemma which we will use in the proof of Theorem 1.27

Lemma 5.3. Let $H_{1}$ be a polynomial semigroup in $\mathcal{G}$ and let $\Gamma$ be a compact family in Poly. Let $H_{2}=\left\langle H_{1}, \Gamma\right\rangle$ be the semigroup generated by $H_{1}$ and $\Gamma$. Suppose

(1) $H_{2} \in \mathcal{G}_{\text {dis }}$ and

(2) $J(\gamma) \cap J_{\min }\left(H_{2}\right)=\emptyset$ for $\gamma \in \Gamma$.

Then $\hat{K}\left(H_{1}\right)=\hat{K}\left(H_{2}\right)$, which then implies $J_{\min }\left(H_{1}\right) \subset J_{\min }\left(H_{2}\right)$ since $\partial \hat{K}\left(H_{1}\right)$ $\subset J_{\min }\left(H_{1}\right)$ and $\partial \hat{K}\left(H_{2}\right) \subset J_{\min }\left(H_{2}\right)$.

Remark 5.4. We recall the facts given in 29, 33] that for any $G \in \mathcal{G}$ we have int $\hat{K}(G)=\hat{K}(G) \cap F(G)$. Moreover, for any $G \in \mathcal{G}_{\text {dis }}$, we have $\operatorname{int} \hat{K}(G) \neq \emptyset$ and $g\left(\hat{K}(G) \cup J_{\min }(G)\right) \subset \operatorname{int} \hat{K}(G)$ for any $g \in G$ such that $J(g) \cap J_{\min }(G)=\emptyset$.

Proof. We begin by first showing that $J_{\min }\left(H_{1}\right) \subset J_{\min }\left(H_{2}\right)$. Let $\mathcal{C}$ be the set of all connected components of $\overline{\bigcup_{\gamma \in \Gamma} J(\gamma)}$. By Lemma 4.8, $M_{1}:=\min _{C \in \mathcal{C}} C$ exists with respect to the surrounding order. Let $J_{1} \in \mathcal{J}_{H_{2}}$ be the element containing $M_{1}$. Let $J_{2} \in \mathcal{J}_{H_{2}}$ be the element containing $J_{\min }\left(H_{1}\right)$. Let $J_{0}:=\min \left\{J_{1}, J_{2}\right\} \in \mathcal{J}_{H_{2}}$. Then for each $g \in H_{1} \cup \Gamma$, either $J_{0}<J(g)$ or $J(g) \subset J_{0}$. By Lemma2.14 and Lemma2.16. 
we obtain that for each $g \in H_{1} \cup \Gamma$, either $g^{-1}\left(J_{0}\right)>J_{0}$ or $g^{-1}\left(J_{0}\right) \subset J_{0}$. By Corollary 2.9 or Theorem 1.14 it follows that $A:=\bigcup_{J \in \mathcal{J}_{H_{2}}, J \geq J_{0}} J$ is closed, $\sharp A \geq 3$, and $g^{-1}(A) \subset A$ for each $g \in H_{2}$. Therefore $J\left(H_{2}\right) \subset A$. Thus $J\left(H_{2}\right)=A$ and hence $J_{0}=J_{\min }\left(H_{2}\right)$. From assumption (2), however, it must be the case that $J_{0}=J_{2}$. Therefore $J_{\min }\left(H_{1}\right) \subset J_{2}=J_{\min }\left(H_{2}\right)$, as desired.

By Remark 5.4 and Theorem 1.20, it follows that for each $\gamma \in \Gamma$, we have $\gamma\left(\partial \hat{K}\left(H_{1}\right)\right) \subset \gamma\left(J_{\min }\left(H_{1}\right)\right) \subset \gamma\left(J_{\min }\left(H_{2}\right)\right) \subset \operatorname{int} \hat{K}\left(H_{2}\right)$. Let $\gamma \in \Gamma$ and let $U$ be any connected component of $\operatorname{int} \hat{K}\left(H_{1}\right)$, which we note is simply connected by the maximum principle. Then $\partial \gamma(U) \subset \gamma(\partial U) \subset$ int $\hat{K}\left(H_{2}\right)$. Let $V$ be the connected component of $\operatorname{int} \hat{K}\left(H_{2}\right)$ containing the connected set $\gamma(\partial U)$. Then $\partial \gamma(U) \subset V$. By the maximum principle, $\overline{\mathbb{C}} \backslash \bar{V}$ is connected and unbounded. Hence $\gamma(U) \subset V$. From this argument, it follows that for each $\gamma \in \Gamma$, we have $\gamma\left(\hat{K}\left(H_{1}\right)\right) \subset \hat{K}\left(H_{2}\right) \subset \hat{K}\left(H_{1}\right)$, where the last inclusion holds since $H_{1} \subset H_{2}$. Thus since $\hat{K}\left(H_{1}\right)$ is forward invariant under each $\gamma \in \Gamma$ and under each map in $H_{1}$, it is also forward invariant under each map in $H_{2}$. We then conclude that $\hat{K}\left(H_{1}\right) \subset \hat{K}\left(H_{2}\right)$, which together with the reverse inclusion already noted gives $\hat{K}\left(H_{1}\right)=\hat{K}\left(H_{2}\right)$.

Definition 5.5. Let $G$ be a rational semigroup and let $N$ be a positive integer. We define $S H_{N}(G)$ to be the set of all $z \in \overline{\mathbb{C}}$ such that there exists a neighborhood $U$ of $z$ such that for all $g \in G$ we have $\operatorname{deg}(g: V \rightarrow U) \leq N$ for each connected component $V$ of $g^{-1}(U)$.

Definition 5.6. Let $G$ be a rational semigroup. We define

$$
U H(G)=\overline{\mathbb{C}} \backslash \bigcup_{N=1}^{\infty} S H_{N}(G)
$$

Remark 5.7. For a rational semigroup $G$ we note that each $S H_{N}(G)$ is open and thus $U H(G)$ is closed.

Remark 5.8. For a rational semigroup $G$ we see that $U H(G) \subset P(G)$. This holds since for $z \notin P(G)$ and $U=B(z, \delta)$ such that $U \cap P(G)=\emptyset$ it must be the case (by an application of the Riemann-Hurwitz relation) that $\operatorname{deg}(g: V \rightarrow U)=1$ for each connected component $V$ of $g^{-1}(U)$.

Remark 5.9. We note from Lemma 1.14 in 22 that the attracting cycles of $g$, the parabolic cycles of $g$, and the boundary of every Siegel disk of $g$ are contained in $U H(\langle g\rangle)$, for any polynomial $g$ with $\operatorname{deg}(g) \geq 2$. Hence we may conclude that such points are also in $U H(G)$ for any $G$ containing $g$.

Proof of Theorem 1.27. Assume the conditions stated in the hypotheses. By the definition of semihyperbolic, our goal is to show $J(G) \subset S H_{K}(G)$ for some $K \in \mathbb{N}$. We will show the equivalent statement that $J(G) \cap U H(G)=\emptyset$. Since $U H(G) \subset$ $P(G)$ and $P^{*}(G) \cap J(G) \subset J_{\min }(G)$, we have only to show $J_{\min }(G) \subset \mathbb{C} \backslash U H(G)$.

By Theorem 1.20 or $\left[29,33\right.$ we know that $\sharp J_{\min }(G) \geq 3$. Thus hypothesis (2) and Lemma2.16 imply $\gamma^{-1}\left(J_{\min }(G)\right) \cap J_{\min }(G)=\emptyset$ for $\gamma \in \Gamma$, which in turn implies (by Lemma 2.14) $\gamma^{-1}\left(J_{\min }(G)\right)>J_{\min }(G)$. Thus, for all $\gamma \in \Gamma$,

$$
\gamma^{-1}(J(G)) \cap A=\emptyset,
$$

where $A=P H\left(J_{\min }\right)$. 
Since $\Gamma$ is compact in Poly, $d=\min _{\gamma \in \Gamma} \operatorname{dist}\left(\gamma^{-1}(J(G)), A\right)>0$. By (II) there exists $d_{1}>0$ such that for all $\gamma \in \Gamma$, for all $z \in J(G)$, and all components $U$ of $\gamma^{-1}\left(B\left(z, d_{1}\right)\right)$ we have

$$
U \cap B(A, d / 2)=\emptyset
$$

Now by Lemma 5.3 and by hypothesis (3) we have $U H(H) \cap \mathbb{C} \subset P^{*}(H) \cap F(H) \subset$ $\hat{K}(H) \cap F(H)=\operatorname{int} \hat{K}(H)=\operatorname{int} \hat{K}(G) \subset F(G)$, and so, taking complements, $J_{\min }(G) \subset \mathbb{C} \backslash U H(H)$.

Claim. There exists $b \in U H(H) \cap \operatorname{int} \hat{K}(H)$.

Proof of the Claim. Lemma 5.3 and Remark 5.4 show that $\operatorname{int} \hat{K}(H)=\operatorname{int} \hat{K}(G) \neq$ $\emptyset$. Let $g_{0} \in H$ and consider the iterates $\left\{g_{0}^{n}\right\}$ at any $w \in \operatorname{int} \hat{K}(H) \subset F(H)$. Hypothesis (3) implies $U H(H) \cap \mathbb{C} \subset F(H)$, which implies that $g_{0}$ cannot have a cycle of Siegel disks or a parabolic cycle (see Remark 5.9). Thus by Sullivan's No Wandering Domains Theorem the orbit $\left\{g_{0}^{n}(w)\right\}$ must be drawn toward an attracting cycle in $\mathbb{C}$. By replacing, if necessary, $g_{0}$ by an iterate we may assume that $g_{0}^{n}(w)$ approaches a finite fixed point $b$ of $g_{0}$. Thus $b \in U H(H) \cap \mathbb{C} \subset P^{*}(H) \cap$ $F(H) \subset \hat{K}(H) \cap F(H)=\operatorname{int} \hat{K}(H)$, which completes the proof of the claim.

Now let $z \in J_{\min }(G) \subset \mathbb{C} \backslash U H(H)$. Then there exists $\delta>0$ such that $B(z, 2 \delta) \subset$ $\mathbb{C} \backslash U H(H)$. Since $g(U H(H)) \subset U H(H)$ for each $g \in H$, we must have $g(b) \notin$ $B(z, 2 \delta)$. Since $H$ is normal at $b$, there exists $\epsilon_{1}>0$ such that $g \in H$ gives $g\left(B\left(b, \epsilon_{1}\right)\right) \cap B(z, \delta)=\emptyset$, which implies $g^{-1}(B(z, \delta)) \cap B\left(b, \epsilon_{1}\right)=\emptyset$. Since $z \in$ $\mathbb{C} \backslash U H(H)$ there exists $\delta_{1}<\delta$ and $N \in \mathbb{N}$ such that for all $h \in H$ and for all components $V$ of $h^{-1}\left(B\left(z, \delta_{1}\right)\right)$ we have $\operatorname{deg}\left(h: V \rightarrow B\left(z, \delta_{1}\right)\right) \leq N$.

Fix $h \in H$ and consider a component $V$ of $h^{-1}\left(B\left(z, \delta_{1}\right)\right)$ and note that the maximum principle implies that $V$ is simply connected. Let $\phi_{V, h}: B(0,1) \rightarrow V$ be the Riemann map chosen such that $h \circ \phi_{V, h}(0)=z$. By applying the distortion lemma, Lemma 1.10 in [22], there exists $0<\delta_{2}<\delta_{1}$ such that the component $W$ of $\left(h \circ \phi_{V, h}\right)^{-1}\left(B\left(z, \delta_{2}\right)\right)$ containing 0 is such that $\operatorname{diam} W \leq c$, where $c>0$ is a small number independent of $h$, to be specified later.

Note that, in the above, the set $V$ does depend on $h \in H$. Yet for each $h \in H$, the set $\phi_{V, h}(B(0,1))=V$ does not meet $B\left(b, \epsilon_{1}\right)$, and so the family $\left\{\phi_{V, h}\right\}_{h \in H}$ is normal on $B(0,1)$. Thus

$$
\operatorname{diam}_{V, h}(W)<d_{1} / 10
$$

when $c$ is sufficiently small.

Let $g \in G$. If $g \in H$, then (since $\left.\delta_{2}<\delta_{1}\right)$ we have $\operatorname{deg}\left(g: V \rightarrow B\left(z, \delta_{2}\right)\right) \leq$ $N$ where $V$ is any component of $g^{-1}\left(B\left(z, \delta_{2}\right)\right)$. If $g \notin H$, then we write $g=$ $h \gamma g_{1}$, where $g_{1} \in G \cup\{i d\}, h \in H \cup\{i d\}$ and $\gamma \in \Gamma$. Let $V_{0}$ be a component of $\gamma^{-1} h^{-1}\left(B\left(z, \delta_{2}\right)\right)$. Thus we have $\operatorname{deg}\left(h \gamma: V_{0} \rightarrow B\left(z, \delta_{2}\right)\right) \leq N M$, where $M=$ $\max _{\gamma \in \Gamma}\{\operatorname{deg} \gamma\}$. By (III) we have $\operatorname{diam} \gamma\left(V_{0}\right)<d_{1} / 10$. By the definition of $d_{1}$ we have $V_{0} \cap B(A, d / 2)=\emptyset$, and thus $V_{0} \cap P(G)=\emptyset$. Using the maximum principle applied to the polynomial $h \gamma$ implies $V_{0}$ is simply connected, and hence each branch of $g_{1}^{-1}$ is well defined on $V_{0}$. So for all components $V_{1}$ of $g^{-1}\left(B\left(z, \delta_{2}\right)\right)$ we have $\operatorname{deg}\left(g: V_{1} \rightarrow B\left(z, \delta_{2}\right)\right) \leq N M$.

In the above, $N$ depends on $z$, but what we have shown is that $z \in J_{\min }(G)$ implies $z \in J_{\min }(G) \cap S H_{N}(H)$ for some $N$, which in turn implies $z \in J_{\min }(G) \cap$ $S H_{N M}(G)$, thus giving $z \notin U H(G)$. 
Proof of Theorem 1.29. The proof follows the same line as the proof of Theorem 1.27 We note that the usual Koebe Distortion Theorem applies (without needing to invoke the distortion Lemma 1.10 in [22]), and on the domains of interest in the proof each $\gamma$ is one-to-one by hypothesis (4) and each $h \in H$ is one-to-one by hypothesis (3). We omit the details.

\section{REFERENCES}

[1] Lars V. Ahlfors. Conformal Invariants: Topics in Geometric Function Theory. McGraw-Hill, New York, 1973. MR0357743 (50:10211)

[2] Alan F. Beardon. Iterations of Rational Functions. Springer-Verlag, New York, 1991. MR.1128089 (92j:30026)

[3] David A. Boyd. The immediate basin of attraction of infinity for polynomial semigroups of finite type. J. London Math. Soc. (2), 69(1):201-213, 2004. MR2025336 (2004j:37081)

[4] Rainer Brück. Geometric properties of Julia sets of the composition of polynomials of the form $z^{2}+c_{n}$. Pacific J. Math., 198(2):347-372, 2001. MR1835513(2002d:37078)

[5] Rainer Brück, Matthias Büger, and Stefan Reitz. Random iterations of polynomials of the form $z^{2}+c_{n}$ : Connectedness of Julia sets. Ergodic Theory Dynam. Systems, 19(5):1221-1231, 1999. MR1721617 (2000h:37064)

[6] Matthias Büger. Self-similarity of Julia sets of the composition of polynomials. Ergodic Theory Dynam. Systems, 17(6):1289-1297, 1997. MR1488318 (98k:58190)

[7] Matthias Büger. On the composition of polynomials of the form $z^{2}+c_{n}$. Math. Ann., 310(4):661-683, 1998. MR 1619744 (99c:30050)

[8] Lennart Carleson and Theodore W. Gamelin. Complex Dynamics. Springer-Verlag, New York, 1993. MR $1230383(94 \mathrm{~h}: 30033)$

[9] John Erik Fornæss and Nessim Sibony. Random iterations of rational functions. Ergodic Theory Dynam. Systems, 11(4):687-708, 1991. MR.1145616 (93c:58173)

[10] Z. Gong and F. Ren. A random dynamical system formed by infinitely many functions. Journal of Fudan University, 35:387-392, 1996. MR1435167 (98k:30032)

[11] A. Hinkkanen and G.J. Martin. The dynamics of semigroups of rational functions I. Proc. London Math. Soc., 3:358-384, 1996. MR1397693 (97e:58198)

[12] S.B. Nadler. Continuum Theory: An introduction. Marcel Dekker, 1992. MR1192552 (93m:54002)

[13] Rich Stankewitz. Completely invariant Julia sets of rational semigroups. Ph.D. thesis, University of Illinois, 1998.

[14] Rich Stankewitz. Completely invariant Julia sets of polynomial semigroups. Proc. Amer. Math. Soc., 127(10):2889-2898, 1999. MR1600149 (2000a:37032)

[15] Rich Stankewitz. Completely invariant sets of normality for rational semigroups. Complex Variables Theory Appl., 40(3):199-210, 2000. MR1753707 (2000m:37069)

[16] Rich Stankewitz. Density of repelling fixed points in the Julia set of a rational or entire semigroup. J. Difference Equ. Appl., 16(5-6):763-771, 2010. MR2675604

[17] Rich Stankewitz, W. Conatser, T. Butz, B. Dean, Y. Li, and K. Hart. JULIA 2.0 Fractal Drawing Program. http://rstankewitz.iweb.bsu.edu/JuliaHelp2.0/Julia.html.

[18] Rich Stankewitz, Toshiyuki Sugawa, and Hiroki Sumi. Some counterexamples in dynamics of rational semigroups. Ann. Acad. Sci. Fenn. Math., 29(2):357-366, 2004. MR2097238 (2005m:37107)

[19] Rich Stankewitz and Hiroki Sumi. Structure of Julia sets of polynomial semigroups with bounded finite postcritical set. Appl. Math. Comput., 187(1):479-488, 2007. MR2323604 (2008d:37081)

[20] Hiroki Sumi. On Hausdorff dimension of Julia sets of hyperbolic rational semigroups. Kodai. Math. J., 21(1):10-28, 1998. MR1625124 (99h:30029)

[21] Hiroki Sumi. Skew product maps related to finitely generated rational semigroups. Nonlinearity, 13:995-1019, 2000. MR/1767945 (2001g:37060)

[22] Hiroki Sumi. Dynamics of sub-hyperbolic and semi-hyperbolic rational semigroups and skew products. Ergod.Th. E Dynam. Sys., 21:563-603, 2001. MR1827119 (2002d:37073a)

[23] Hiroki Sumi. Dimensions of Julia sets of expanding rational semigroups. Kodai Mathematical Journal, 28(2):390-422, 2005. MR2153926 (2006c:37050) 
[24] Hiroki Sumi. Semi-hyperbolic fibered rational maps and rational semigroups. Ergod.Th. 8 Dynam. Sys., 26:893-922, 2006. MR 2237476(2007d:37068)

[25] Hiroki Sumi. Interaction cohomology of forward or backward self-similar systems. Adv. Math., 222:729-781, 1009.

[26] Hiroki Sumi. Dynamics of polynomial semigroups with bounded postcritical set in the plane. RIMS Kokyuroku, 1447:198-215, 2005. (Proceedings paper.)

[27] Hiroki Sumi. Dynamics of postcritically bounded polynomial semigroups and interaction cohomology. RIMS Kokyuroku, 1447:227-238, 2005. (Proceedings paper.)

[28] Hiroki Sumi. Random dynamics of polynomials and devil's staircase-like functions in the complex plane. Appl. Math. Comput., 187:489-500, 2007. (Proceedings paper.) MR 2323605 (2008d:37075)

[29] Hiroki Sumi. Dynamics of postcritically bounded polynomial semigroups I: connected components of the Julia sets. Discrete and Continuous Dynamical Systems Series A, Vol. 29, No. 3, 2011, 1205-1244.

[30] Hiroki Sumi. Dynamics of postcritically bounded polynomial semigroups II: fiberwise dynamics and the Julia sets. Preprint 2008, http://arxiv.org/abs/1007.0613.

[31] Hiroki Sumi. Dynamics of postcritically bounded polynomial semigroups III: classification of semi-hyperbolic semigroups and random Julia sets which are Jordan curves but not quasicircles. Ergodic Theory Dynam. Systems (2010), 30, No. 6, 1869-1902. MR 2736899

[32] Hiroki Sumi. Random complex dynamics and semigroups of holomorphic maps. Proc. London Math. Soc. (2011), 102 (1), 50-112. MR2747724

[33] Hiroki Sumi. Dynamics of postcritically bounded polynomial semigroups. Preprint 2006, http://arxiv.org/abs/math.DS/0703591.

[34] Random complex dynamics and devil's coliseums. Preprint 2011, http://arxiv.org/abs/ 1104.3640.

[35] Hiroki Sumi and Mariusz Urbański. The equilibrium states for semigroups of rational maps. Monatsh. Math. 156(4):371-390, 2009. MR2486604

[36] Hiroki Sumi and Mariusz Urbański. Real analyticity of Hausdorff dimension for expanding rational semigroups. Ergod. Th. ES Dynam. Sys. 30(2):601-633, 2010. MR2599895

[37] Hiroki Sumi and Mariusz Urbański. Measures and dimensions of Julia sets of semi-hyperbolic rational semigroups. Discrete and Continuous Dynamical Systems Ser. A., Vol. 30, No. 1, 2011, 313-363.

[38] Y. Sun and C-C. Yang. On the connectivity of the Julia set of a finitely generated rational semigroup. Proc. Amer. Math. Soc., 130(1):49-52, 2001. MR1855618 (2002e:37065)

[39] W. Zhou and F. Ren. The Julia sets of the random iteration of rational functions. Chinese Sci. Bulletin, 37(12):969-971, 1992.

Department of Mathematical Sciences, Ball State University, Muncie, Indiana 47306

E-mail address: rstankewitz@bsu.edu

Department of Mathematics, Graduate School of Science, Osaka University, 1-1, Machikaneyama, Toyonaka, Osaka, 560-0043, Japan

E-mail address: sumi@math.sci.osaka-u.ac.jp 